\title{
gु \\ Dynamics of weakly coupled parametrically forced oscillators
}

\author{
P. Salgado Sánchez, J. Porter, ${ }^{*}$ I. Tinao, and A. Laverón-Simavilla \\ Escuela Técnica Superior de Ingeniería Aeronáutica y del Espacio, Universidad Politécnica de Madrid, \\ Plaza de Cardenal Cisneros 3, 28040 Madrid, Spain
}

(Received 27 November 2015; revised manuscript received 16 June 2016; published 29 August 2016)

\begin{abstract}
The dynamics of two weakly coupled parametric oscillators are studied in the neighborhood of the primary subharmonic instability. The nature of both primary and secondary instabilities depends in a critical way on the permutation symmetries, if any, that remain after coupling is considered, and this depends on the relative phases of the parametric forcing terms. Detailed bifurcation sets, revealing a complex series of transitions organized in part by Bogdanov-Takens points, are calculated for representative sets of parameters. In the particular case of out-of-phase forcing the predictions of the coupled oscillator model are compared with direct numerical simulations and with recent experiments on modulated cross waves. Both the initial Hopf bifurcation and the subsequent saddle-node heteroclinic bifurcation are confirmed.
\end{abstract}

DOI: 10.1103/PhysRevE.94.022216

\section{INTRODUCTION}

Oscillations arise in an enormous variety of systems, from basic mechanical devices like a mass on a spring, to surface or pressure waves in continuous media, to population [1] and climate [2] cycles in the natural environment. In dissipative systems such periodic variation typically involves both a restoring force, which determines the frequency of natural oscillations, and a driving force, which sustains them against energy losses. If the driving mechanism depends on time, its interaction with the restoring force can lead to interesting resonance phenomena. Further possibilities for resonance arise if the oscillatory system is not isolated, but interacts with other dynamical systems. In particular, there has been considerable recent interest in how an oscillatory system couples to other identical (or similar) systems and the collective behaviors that ensue.

The study of coupled oscillators, which can be traced to Huygens' work with pendulum clocks in the 17th century [3], is concerned with collective phenomena such as synchronization, phase locking, and amplitude death. These features, and others, depend both on the individual dynamics of the component oscillators and the properties of the coupling between them.

In many cases it is reasonable to assume that the oscillators are equivalent in the absence of coupling: identical fireflies, for example, in a population undergoing synchronous flashing [4]. This permutation symmetry between individual oscillators may or may not persist when coupling is included, and the extent to which it does is a key factor in determining the structure of invariant subspaces and the possible classes of solutions. The type of coupling that applies (global all-to-all, nonlocal, local, etc.) plays a central role in determining the residual exchange symmetry.

The type of forcing mechanism that drives individual oscillations has an important effect as well, particularly with regard to phase locking. If the forcing mechanism is independent of time (like a steady wind blowing waves across a water surface), then the response of the system will inherit this time translation invariance and there will be no preferred phase of

\footnotetext{
*jeff.porter@upm.es
}

the uncoupled oscillation. Collections of such oscillators can, in the limit of weak coupling, be reduced to phase models [5] where each oscillator is influenced only by the phase (not amplitude) of the other oscillators that are coupled to it.

If individual oscillations occur in response to periodic forcing, then, because there is no continuous time translation symmetry, phases in the uncoupled problem are not equivalent. Each oscillator has (one or more) preferred phases that will be selected when coupling is weak; in this limit, synchronization depends not on coupling but on the relationship between the individual forcing functions. If the forcing is direct in character (i.e., it excites synchronous oscillations for any forcing amplitude), then a typical oscillator will have, in the absence of coupling, a single preferred phase. If, on the other hand, the time-dependent forcing is parametric in nature, then the typical response is subharmonic, at half the forcing frequency, and there will be (at least) two preferred phases related by time translation through one forcing period. Among other things, this means that collections of weakly coupled parametrically forced oscillators have more possible solutions than their directly forced counterparts and hence the potential for more complicated dynamics.

Coupled parametrically forced oscillators have received less attention than autonomous or directly forced systems despite potential applications in microelectromechanical systems (MEMS) [6,7] and optical parametric oscillators [8], for example. In this paper, we are particularly interested in the following application: the dynamics of parametrically excited surface waves such as cross waves [9]. These subharmonic waves occur regularly in wavemaker experiments with a vibrating surface or boundary [10-14]. Horizontally vibrated containers of fluid, in particular, are often used to study surface waves [15-18] and, if the container is relatively large compared to the decay length of the waves, then the excitation patterns generated at the left and right endwalls (or wavemakers) can be considered to be weakly interacting. This is the situation in several recent experiments $[19,20]$.

There are a number of studies of coupled parametrically forced oscillators in the literature to which the current paper is related. Bena and Van den Broeck [21] considered a large collection of linear oscillators in the mean-field limit driven by square-wave excitation with randomly distributed phases 
for each oscillator. They found complex stability boundaries for collective behavior with a wider region of instability than for individual parametric excitation.

Goldobin and Pikovsky [22] studied the collective excitations in a population of globally coupled weakly nonlinear oscillators parametrically driven by sinusoidal forcing, with uniformly distributed phases. A traveling wave regime was found, as was, in general, a primary instability that could be either steady or oscillatory.

Copelli and Lindenberg [23] studied a pair of linear oscillators driven by piecewise constant (square-wave) parametric forcing and found complicated stability boundaries with sensitive dependence on the phase difference between the two forcing functions. They observed that collective behavior (i.e., similarity to mean-field models describing many oscillators) is reflected to a greater extent in the dimer with out-of-phase forcing; an analytical solution for this case was presented in [24].

In the context of bifurcation theory, the interaction of two parametrically forced oscillators, which each undergo steady state (pitchfork) bifurcations in the absence of coupling, is associated with the unfolding of a double-zero bifurcation. This was studied, with both Huygens (exchange) symmetry and odd-Huygens symmetry by Kitanov et al. [25]. In the case of odd-Huygens symmetry, which is the relevant one for subharmonic modes like those considered here, both primary and secondary pitchfork bifurcations were found, as were, with certain unfolding parameters, Hopf and heteroclinic bifurcations.

Danzl and Moehlis [26] considered networks of nonlinear parametrically forced oscillators with weak coupling, used the implicit function theorem to prove the persistence of the uncoupled solution classes, and investigated this numerically. Far from the weak coupling limit, interesting dynamics such as antisynchronized chaotic behavior were found.

The current paper shares with [26] an emphasis on symmetries and the assumption of weak coupling and with [23,24] an interest in the effect of the forcing phases. As in [23], we utilize a bifurcation theory (unfolding) approach. The principal contributions of the current paper are as follows.

In Sec. II, we briefly review the dynamics of a single parametrically forced oscillator in the neighborhood of the dominant subharmonic instability and note the secondary Hopf and global bifurcations that can occur with a nonconservative nonlinear term. We consider a pair of coupled oscillators in Sec. IV. Here the residual symmetry is critical in organizing the structure of solutions near the initial instability where the hyperbolicity assumption of [26] fails. Unlike in [23,24], we do not calculate multiple resonance tongue boundaries, but instead provide a complete bifurcation picture for the weakly nonlinear problem in a neighborhood of the dominant subharmonic instability; this includes secondary local and global bifurcations, and organizing Bogdanov-Takens points. Bifurcation sets are calculated as a function of forcing and detuning for representative sets of parameters. The detailed bifurcation scenario obtained for two coupled oscillators is compared, to the extent possible, with the recent experiments of [20] in Sec. V and with direct numerical simulations of a corresponding two-dimensional vibrated fluid system in Sec. VI. Conclusions are presented in Sec. VII.

\section{DYNAMICS OF A PARAMETRICALLY FORCED OSCILLATOR}

A wide range of parametrically forced oscillators can be approximated by a damped nonlinear Mathieu equation of the form

$$
\ddot{x}+2 \gamma \dot{x}+[1+4 f \cos (2 \omega t-\phi)] x+G(x, \dot{x})=0,
$$

where $\gamma>0$ is the damping coefficient, $f>0$ is the forcing amplitude, $G(x, \dot{x})$ denotes nonlinear terms, and time has been scaled with the natural frequency of the oscillator. For a single oscillator the phase $\phi$ is of no consequence and can be removed by a simple shift in time, but it is left in Eq. (1) to anticipate the general case of coupled oscillators.

It is well known that the linear stability problem leads to a series of resonance tongues $[27,28]$ centered at integer values of $\omega$. We assume here that the system is weakly damped and near the primary subharmonic $(\omega=1)$ instability by writing $\omega=1+v$. Using a perturbation expansion of the form

$$
x(t)=A(t) e^{-i(\omega t+\pi / 4)}+\text { c.c. }+\cdots,
$$

where c.c. denotes the complex conjugate, leads to the amplitude equation

$$
\dot{A}=(-\gamma+i \nu) A+f e^{i \phi} \bar{A}-\left(a_{r}+i a_{i}\right)|A|^{2} A+\cdots,
$$

which applies when $|A|, \gamma, f,|\nu| \ll 1$. If when $\gamma=f=0$ the nonlinearity $G(x, \dot{x})=G(x)$ is conservative, then the cubic coefficient is imaginary at leading order and $a_{r}=0$.

Equation (3) can be simplified with the rescaling

$$
t \rightarrow t / \gamma, \quad(f, v) \rightarrow(f, v) \gamma, \quad A \rightarrow \sqrt{\frac{\gamma}{\left|a_{i}\right|}} A,
$$

and, if $a_{i}<0$, the additional substitutions $A \rightarrow \bar{A}$ and $(v, \phi) \rightarrow-(v, \phi)$,

$$
\dot{A}=(-1+i v) A+f e^{i \phi} \bar{A}-(a+i)|A|^{2} A+\cdots,
$$

where $a=a_{r} /\left|a_{i}\right|$.

The primary subharmonic instability of a parametrically forced oscillator is a pitchfork bifurcation that produces two symmetry-related steady states $\pm A_{s}$. This bifurcation occurs along the curve

$$
\text { pitchfork : } f^{2}=1+v^{2} \text {. }
$$

Since the nontrivial steady states $A_{s}$ satisfy

$$
\left|A_{s}\right|^{2}=\frac{v-a}{a^{2}+1} \pm \sqrt{\frac{f^{2}}{a^{2}+1}-\left(\frac{1+a v}{a^{2}+1}\right)^{2}},
$$

the primary bifurcation is supercritical if $v<a$ and subcritical if $v>a$. In the subcritical case, the initially unstable steady branch is stabilized in a saddle-node bifurcation along the half-line,

$$
\text { saddle-node : } f^{2}=\frac{(1+a v)^{2}}{1+a^{2}} ; v>a .
$$

These two bifurcation sets are illustrated in Fig. 1 for the case $a=0$ corresponding to conservative nonlinearity. In this case there are no additional bifurcations, nor are there any with dissipative nonlinearity $a>0$, although the saddle-node bifurcation is delayed in that case. 


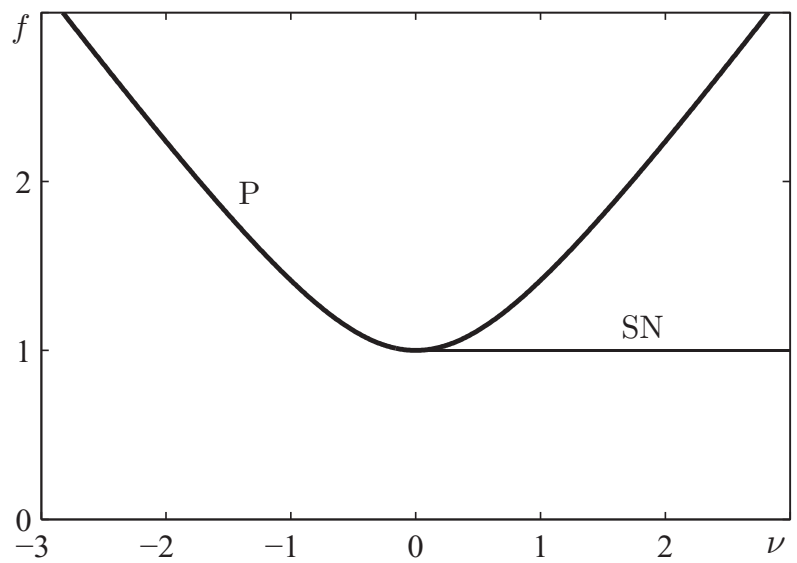

FIG. 1. Pitchfork (P, thick curve) and saddle-node ( $\mathrm{SN}$, thin curve) bifurcation sets for a single parametrically forced oscillator in the case of conservative nonlinearity $a=0$.

The case $a<0$, however, is qualitatively different because the nonlinearity can provide energy to the oscillator and sustain motion, even without parametric forcing. With $f=0$, Eq. (5) contains an unstable rotating solution with constant amplitude $|A|=\sqrt{-1 / a}$ and frequency $v+1 / a$. Furthermore, the two (symmetry-related) stable steady states created in a pitchfork bifurcation for $v<a$ and in a saddle-node bifurcation for $v>a$ undergo Hopf bifurcation along the curve

$$
\text { Hopf : } f^{2}=\frac{1}{4}+\left(\frac{1+2 a v}{2 a}\right)^{2} ; \quad v<\frac{a^{2}-1}{2 a} .
$$

This Hopf bifurcation set terminates at a Bogdanov-Takens point when it intersects the line of saddle-node bifurcations:

$$
\text { Bogdanov-Takens : } f=\frac{\sqrt{1+a^{2}}}{2} ; \quad v=\frac{a^{2}-1}{2 a} \text {. }
$$

The Hopf bifurcation occurs when the stable steady state branch [Eq. (7) with the "+" sign] reaches a fixed amplitude $A_{H}$, independent of $v$,

$$
\left|A_{H}\right|^{2}=-\frac{1}{2 a}
$$

while the Hopf frequency $\omega_{H}$ increases with distance from the Bogdanov-Takens point,

$$
\omega_{H}=\sqrt{\frac{2}{a}\left(v+\frac{1-a^{2}}{2 a}\right)} .
$$

The normal form (first Lyapunov) coefficient that determines whether the Hopf bifurcation is supercritical or subcritical can be calculated following [29], for example, and is found to be negative throughout the region of existence; contours of this coefficient are shown in Fig. 2. This secondary Hopf bifurcation is therefore always supercritical. The stable modulated states generated here grow in amplitude and are destroyed by a homoclinic bifurcation. As shown in Fig. 3, the nature of this homoclinic bifurcation changes with the stability of the trivial state. If $f^{2}>1+v^{2}$ the trivial state is unstable and the periodic modulated states collide with it in a homoclinic gluing bifurcation. This gluing bifurcation transforms the symmetry-related pair of stable modulated

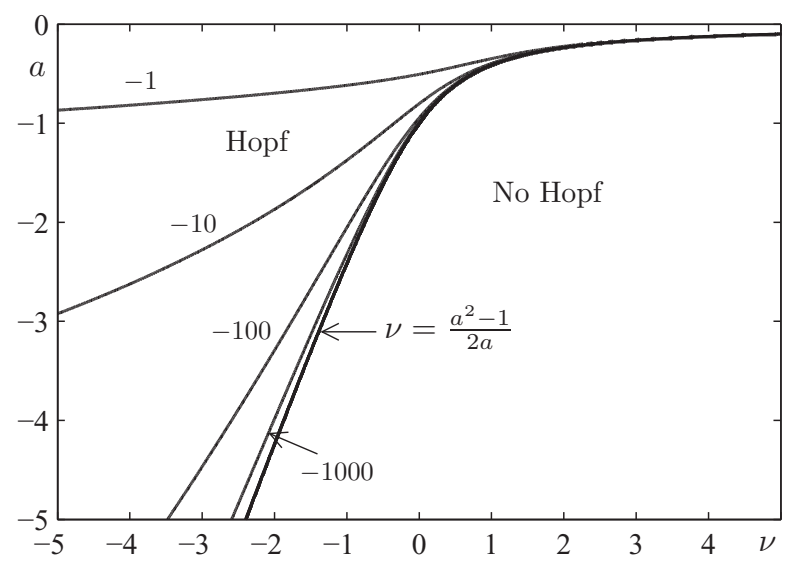

FIG. 2. Contours of the normal form (first Lyapunov) coefficient $l_{1}$ (see [29]) in the $(v, a)$ plane. This coefficient is always negative, showing that the Hopf bifurcation, which occurs for $v<\left(a^{2}-\right.$ $1) /(2 a)$, is supercritical.

states into the unique rotating (unbounded phase) state, the one supported at $f=0$ purely by nonlinearity. Note that the stable rotating branch created in the gluing bifurcation soon undergoes a saddle-node bifurcation that renders it unstable (as it is for most parameters).

For forcing below the primary pitchfork bifurcation, $f^{2} \leqslant$ $1+v^{2}$, the trivial state is stable and cannot participate in a global bifurcation. The gluing bifurcation therefore splits when crossing below the (subcritical) pitchfork bifurcation set (see Fig. 3) into two separate global bifurcations: a (nonsymmetric) homoclinic bifurcation where the modulated states created in the Hopf bifurcation separately collide with the finite-amplitude saddle fixed points created in the subcritical pitchfork bifurcation and a heteroclinic bifurcation, at slightly larger $f$, where the rotating periodic state

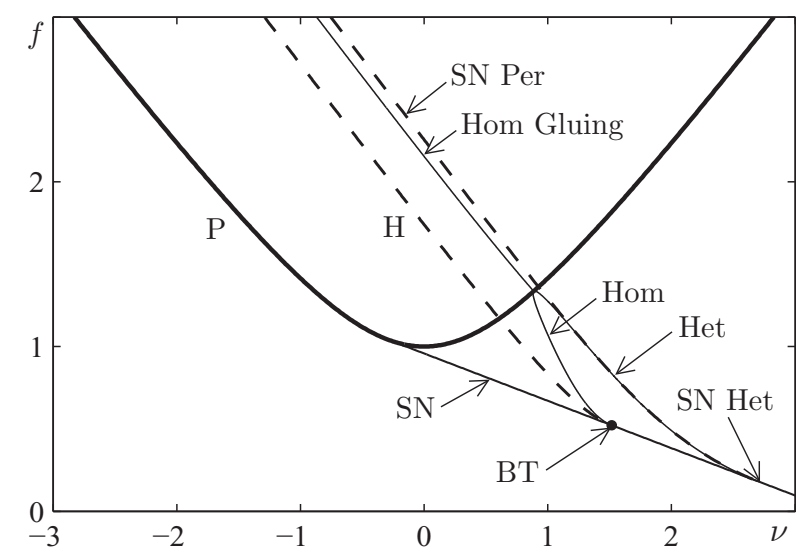

FIG. 3. Bifurcation sets in the case of $a=-0.3$ : pitchfork (P, thick solid curve), saddle-node ( $\mathrm{SN}$, thin solid line), Hopf $(\mathrm{H}$, lower dashed curve), saddle-node of periodic orbits (SN Per, upper dashed curve), homoclinic gluing (Hom Gluing, thin solid curve above pitchfork), homoclinic (Hom, lower thin solid curve below pitchfork), heteroclinic (Het, upper thin solid curve below pitchfork), saddle-node heteroclinic (SN Het), and Bogdanov-Takens point (BT, solid dot). 
simultaneously collides with these same two nontrivial steady states. This heteroclinic bifurcation set eventually merges with the saddle-node bifurcation set to create (for $v>2.67$ ) a saddle-node heteroclinic bifurcation, i.e., a heteroclinic cycle connecting nonhyperbolic stationary solutions with a simple zero eigenvalue (see, e.g., [29]). We note that this is a symmetric version of what is also known as Shilnikov's saddle-node bifurcation [30], saddle-node on invariant circle (SNIC) bifurcation, or saddle-node infinite period (SNIPER) bifurcation. We refer to the usual asymmetric case as a saddle-node homoclinic bifurcation (following the terminology of [29]).

Figure 4 shows one bifurcation diagram obtained with $v=-0.5$, when the primary pitchfork bifurcation is supercritical, and one obtained with $v=1$, when this bifurcation is subcritical. For each of these detuning values, a supercritical Hopf bifurcation occurs on the stable nontrivial steady solution branch. With $v=-0.5$ the periodic modulated states $P_{m}$ created here collide with the origin in a gluing bifurcation at $f=2.678$ that creates a periodic rotating state $P_{r}$. For $v=1$, in contrast, there are distinct homoclinic and heteroclinic bifurcations involving the small-amplitude unstable steady states. Regardless of the detuning value, when $a<0$ stable solutions can only be found relatively close to onset (and for initial conditions not too large); otherwise (most) trajectories diverge due to the destabilizing nonlinearity considered. In this case, a more realistic version of Eq. (5) would include stabilizing fifth-order terms.

\section{WEAKLY COUPLED PARAMETRICALLY FORCED OSCILLATORS}

If two or more parametrically forced oscillators are coupled together, the dynamics is potentially much richer than that described above. This dynamics depends on both the coupling between the oscillators and the individual forcing terms, which act to select particular phases for each oscillator. Motivated by its relevance to recent experiments on horizontally vibrated fluids [20] (see Sec. V), we consider below the case of two coupled oscillators in detail. Since the symmetry arguments are quite general, however, we first describe these in a broader context.

A collection of $n$ linearly coupled parametrically forced oscillators can be described by the equation

$$
\dot{\boldsymbol{A}}=\mathcal{L} \boldsymbol{A}+f \Phi \overline{\boldsymbol{A}}-\mathcal{G}\left(|\boldsymbol{A}|^{2}\right) \boldsymbol{A}+\Lambda \boldsymbol{A},
$$

where $\boldsymbol{A}=\left(A_{1}, A_{2}, \ldots, A_{n}\right)^{T}$. The diagonal matrices $\mathcal{L}, \Phi$, and $\mathcal{G}$ represent the uncoupled problem of Eq. (5): $\mathcal{L}_{j j}=$ $-1+i \nu, \Phi_{j j}=e^{i \phi_{j}}, \mathcal{G}_{j j}=(a+i)\left|A_{j}\right|^{2}$. The coupling matrix $\Lambda$ is assumed to be symmetric (the $j$ th and $k$ th oscillators affect each other in the same way) and we further assume that the forcing differs only in phase (i.e., each oscillator loses stability at the same critical value of the bifurcation parameter $f$ ).

\section{A. General symmetry considerations}

If there is no coupling, $\Lambda=0$, each of the oscillator equations is individually equivariant under reflection $A_{j} \rightarrow$ $-A_{j}$ due to the subharmonic nature of the critical modes. In addition, the identical oscillators may be exchanged, or

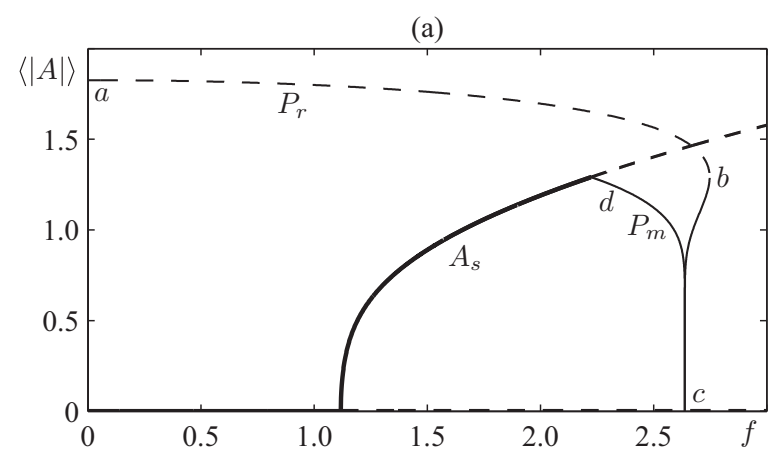

(b)
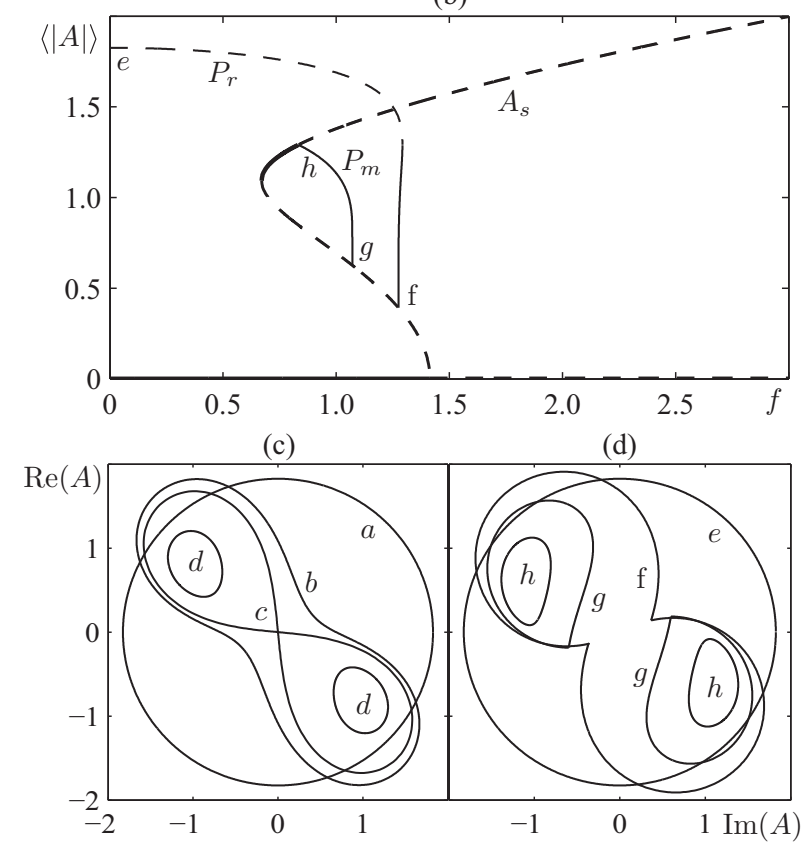

FIG. 4. Bifurcation diagrams for $a=-0.3$ and (a) $v=-0.5$ and (b) $v=1$, showing the (time-averaged) norm of the nontrivial steady states $\left(A_{s}\right)$, periodic modulated solutions $\left(P_{m}\right)$, and periodic rotating solution $\left(P_{r}\right)$. Solid (dashed) curves denote stable (unstable) solutions. With increasing $v$, the primary bifurcation transitions from supercritical to subcritical and the gluing bifurcation splits into distinct homoclinic and heteroclinic bifurcations. A selection of periodic orbits at particular points labeled $a-h$ in these diagrams are shown in (c) and (d), respectively. $a, e$, rotating orbit at $f=0 ; b$, limit point at $f=2.747 ; c$, symmetric homoclinic orbit at $f=2.678 ; d$, small modulated orbits at $f=2.3 ; f$, heteroclinic orbit at $f=1.275$; $g$, homoclinic orbits at $f=1.071 ; h$, small modulated orbits at $f=0.9$.

permuted, in $n$ ! distinct ways. These permutations form a representation of the symmetric group $\boldsymbol{S}_{n}$. For example, the $j$ th and $k$ th oscillators can be exchanged with the operation

$$
\left(A_{j}, A_{k}\right) \rightarrow\left(e^{i \delta_{j k}} A_{k}, e^{-i \delta_{j k}} A_{j}\right),
$$

where $\delta_{j k}=\left(\phi_{j}-\phi_{k}\right) / 2=-\delta_{k j}$. Given a set of possible limit sets for each oscillator (trivial state, stable steady states, unstable steady states, periodic orbits, etc.), there is a larger set of solution classes formed by different combinations of these individual oscillator states. The members of each symmetry (isotropy) class are related by a combination of reflections and permutations. 
As discussed in Danzl and Moehlis [26], the implicit function theorem guarantees that these various uncoupled solution branches persist with the same stability properties in the presence of sufficiently weak coupling, provided they are hyperbolic. Bifurcation points, however, where solutions are nonhyperbolic, will be strongly influenced by the nature of the residual symmetry of the coupled problem and this includes the primary bifurcation, which not only can be shifted by the coupling terms, but may also be transformed from steady to oscillatory.

Coupling breaks the symmetry of the uncoupled problem for two reasons. First, unless the coupling is trivial $(\Lambda=0)$ or uniform all-to-all, it will not commute with all permutations of the oscillators even in the case of identical forcing phases $(\Phi \propto \mathbb{1})$. Second, a nontrivial set of phase differences $\left(\phi_{j} \neq \phi_{k}\right.$ for some $\left.j, k\right)$ means that exchanging oscillators while preserving the parametric forcing terms requires a phase shift as in Eq. (14); $\Phi$ only commutes with ordinary permutations if $\Phi \propto \mathbb{1}$. The phase matrix $\Phi$ can be formally eliminated with the transformation $A_{j} \rightarrow e^{i \phi_{j} / 2} A_{j}$ but this, in general, introduces asymmetric phase shifts into the coupling matrix $\Lambda$. Generally, whether or not a subset of exchange symmetries is preserved by the coupling depends both on the structure of the coupling matrix $\Lambda$ and on the choice of forcing phases $\phi_{j}$.

The case of maximum symmetry occurs with uniform all-to-all coupling and identical forcing phases. In this case, arbitrary permutations are permitted, although the subharmonic reflection symmetry can only be applied collectively with

$$
R: \boldsymbol{A} \rightarrow-\boldsymbol{A} .
$$

This case, with the maximum number of exchange (permutation) symmetries and corresponding fixed point subspaces, is most similar to the uncoupled problem.

The case of minimum symmetry occurs if the coupling and/or forcing phases are general enough that no permutation symmetries remain. The only symmetry is then the basic subharmonic reflection (15).

\section{B. Motivation from vibrated fluid experiments}

Vibrated fluid experiments can produce a wide range of surface wave patterns $[9,31]$, some of the most studied being due to a parametric forcing mechanism [32,33]. Vertically forced Faraday waves, which are (usually assumed to be) excited uniformly throughout the container, cannot easily be described as a system of weakly coupled oscillators. Cross waves, on the other hand, whether driven by wavemakers or by direct (horizontal) motion of a rigid container, are excited by a spatially nonuniform oscillatory field $[19,20,34]$ that can, in many cases, be considered localized. If more than one wavemaker is used, the situation may naturally arise where different parametrically forced surface wave patterns interact "weakly" in the far-field (subcritically forced) region of the surface or interface.

If, for example, two identical wavemakers are spatially separated and driven in phase, then the surface wave patterns they excite after a subharmonic instability may be approximated as weakly coupled parametrically forced oscillators with synchronized forcing. If, instead, these same two wavemakers are driven with distinct phases, then the coupled system behaves differently (it is equivalent to introducing asymmetry in the coupling between the two patterns/oscillators). We note that this second situation automatically occurs when the subharmonic surface waves are excited by rigid horizontal motion of an open fluid container $[19,35,36]$. In this case, while the left endwall is moving into the fluid (leading to upward displacement near the surface), the right endwall is pulling away from the fluid (causing downward displacement near the surface). If the aspect ratio is large enough (the patterns are sufficiently localized), such a system may be approximated by two weakly coupled parametrically forced oscillators with out-of-phase forcing. Each of the two cases mentioned above are considered in detail in the following section, and the case of out-of-phase forcing is compared to recent experimental results in Sec. V and to two-dimensional Navier-Stokes simulations in Sec. VI. To the best of our knowledge, there are no experiments to compare with in the case of synchronous forcing, or more general forcing phase difference, although it is easy to imagine a fluid experiment with independent wavemakers exciting localized cross waves that could test the predictions of the following section in these cases too. We may undertake such an experiment in the near future.

\section{TWO COUPLED PARAMETRICALLY FORCED OSCILLATORS}

We now consider in detail the case of two weakly coupled parametrically forced oscillators. This situation constitutes the simplest interaction possible and allows a relatively complete analysis. As mentioned above, and discussed in more detail in Sec. V, it is relevant to experiments on horizontally vibrated fluids in rigid containers $[19,20]$. In this system, the parametric forcing mechanism [34-36] is concentrated near opposing boundaries (endwalls or wavemakers) and, in containers of sufficiently large aspect ratio, this leads to relatively isolated patterns of subharmonic surface waves that can be considered as weakly interacting.

The governing equations for the complex amplitudes, $A$ and $B$, characterizing the two oscillators are

$$
\begin{aligned}
& \dot{A}=(-1+i v) A+f \bar{A}-(a+i)|A|^{2} A+\mu B, \\
& \dot{B}=(-1+i v) B+f e^{i \phi} \bar{B}-(a+i)|B|^{2} B+\mu A,
\end{aligned}
$$

where the forcing phase of the first equation has, without loss of generality, been set to zero.

It was noted earlier that if the oscillator is affected only by conservative nonlinearities, then the nonlinear coefficient is purely imaginary $(a=0)$. The complex coupling coefficient $\mu=\mu_{r}+i \mu_{i}$ similarly splits into conservative and nonconservative parts, as seen with the energy function

$$
E=\frac{1}{2}\left(|A|^{2}+|B|^{2}\right),
$$

which satisfies

$$
\begin{aligned}
\dot{E}= & -\left(|A|^{2}+|B|^{2}\right)-a\left(|A|^{4}+|B|^{4}\right) \\
& +f \operatorname{Re}\left(A^{2}+e^{-i \phi} B^{2}\right)+2 \mu_{r} \operatorname{Re}(A \bar{B}) .
\end{aligned}
$$

The first two terms reflect linear and nonlinear dissipation, respectively, while the forcing may provide or extract energy 
depending on the phases of $A$ and $B$. The final term shows that only the real part $\mu_{r}$ of the coupling term can affect the energy $E$, while the imaginary part $\mu_{i}$ is conservative. The sign of the contribution to $\dot{E}$ from the coupling depends on the phase of $A \bar{B}$.

\section{A. Symmetries}

Uncoupled problem. Due to the odd character of the critical subharmonic modes under translation through one period of the forcing, the uncoupled problem possesses a separate reflection symmetry for each oscillator,

$$
R_{A}: A \rightarrow-A, \quad R_{B}: B \rightarrow-B .
$$

Permutation symmetry with two oscillators is represented by the single operation,

$$
\kappa:(A, B) \rightarrow\left(e^{-i \phi / 2} B, e^{i \phi / 2} A\right) .
$$

These symmetries of the uncoupled problem generate a representation of the dihedral group (of order eight) $\boldsymbol{D}_{4}$; one may take as generators, for example, $R_{A}$ and $\rho=R_{A} \kappa$.

Coupled problem. Coupling implies that the modes can no longer be reflected independently and only the single (subharmonic) reflection symmetry holds:

$$
R:(A, B) \rightarrow-(A, B) .
$$

Since a pair of (identical) oscillators is described by a single coupling coefficient, the residual symmetry of the coupled problem depends entirely on the phase difference $\phi$. It is easy to show that only two choices of $\phi$ permit any type of permutation symmetry.

Symmetric forcing. If $\phi=0$, there is an interchange symmetry,

$$
\kappa_{0}:(A, B) \rightarrow(B, A),
$$

which, together with $R$, generates the group $Z_{2} \times Z_{2}$. There are two invariant subspaces,

$$
\begin{aligned}
\operatorname{Fix}\left(\kappa_{0}\right) & =\{(A, B) \mid A=B\}, \\
\operatorname{Fix}\left(\kappa_{0} R\right) & =\{(A, B) \mid A=-B\},
\end{aligned}
$$

in which the system is equivalent to a single oscillator.

Antisymmetric forcing. If $\phi=\pi$, there is a cyclic exchange symmetry generated by

$$
\rho:(A, B) \rightarrow i(B, A) .
$$

The corresponding symmetry group, $\boldsymbol{C}_{4}$, has no nontrivial fixed point subspaces. Note that $\rho^{2}=R$.

General forcing. If $\phi \neq 0, \pi$ then the only symmetry is the reflection $R$, representing the group $\boldsymbol{Z}_{\mathbf{2}}$.

Table I summarizes the three ways, depending on phase, that coupling breaks the $\boldsymbol{D}_{4}$ symmetry of the uncoupled problem.

\section{B. Parameter symmetries}

Parameter symmetries can be used to reduce the range of parameters that needs to be considered.

The system (16) is equivariant under the transformation

$$
(A, B) \rightarrow e^{i \phi / 2}(B, A), \quad \phi \rightarrow-\phi,
$$

TABLE I. Diagram showing the three ways that the $\boldsymbol{D}_{\mathbf{4}}$ symmetry of the uncoupled problem is broken for $\mu \neq 0$.

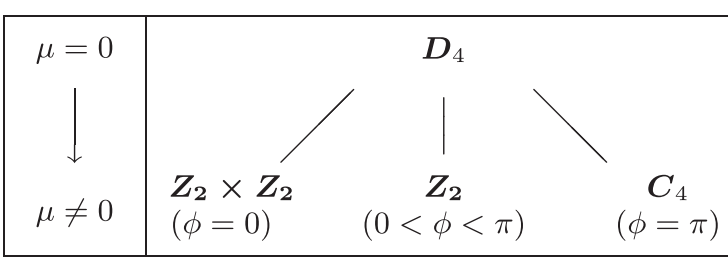

which means that $\phi$ can be restricted, without loss of generality, to $0 \leqslant \phi \leqslant \pi$. Physically, this symmetry reflects the fact that the sign of $\phi$, which determines whether the parametric forcing of $A$ is leading or lagging that of $B$, is not important. Equivalent solutions exist for $\phi \rightarrow-\phi$ and an appropriately phase shifted exchange of $A$ and $B$.

The equivariance of Eqs. (16) under the parameter symmetry

$$
A \rightarrow-A, \quad \mu \rightarrow-\mu
$$

means that $\mu$ can be restricted, without loss of generality, to $\mu_{r}>0$.

\section{Bifurcation analysis}

As shown above, the manner in which the $\boldsymbol{D}_{4}$ symmetry of a pair of identical uncoupled parametrically forced oscillators is broken by (symmetric) coupling depends only on the relative phase of the parametric forcing at each oscillator. We consider now in more detail the three distinct cases.

The coupling coefficient $\mu$ is assumed to be small but, at the same time, we use illustrative values that are large enough to clearly show the different behavior in each case. Specifically, we choose $\mu=0.2 i$ or $\mu=0.05+0.2 i$ for most of the calculations below, electing $\mu_{r} \ll \mu_{i}$ for a nearly conservative coupling term. The bifurcation sets, when not available analytically, are obtained numerically [37].

\section{Symmetric forcing, $\phi=0$}

In this case the equations governing the two subharmonic modes $A$ and $B$ are

$$
\begin{aligned}
& \dot{A}=(-1+i v) A+f \bar{A}-(a+i)|A|^{2} A+\mu B, \\
& \dot{B}=(-1+i v) B+f \bar{B}-(a+i)|B|^{2} B+\mu A
\end{aligned}
$$

Recall that the exchange symmetry $\kappa_{0}:(A, B) \rightarrow(B, A)$ and subharmonic symmetry $R:(A, B) \rightarrow-(A, B)$ generate a representation of the group $\boldsymbol{Z}_{2} \times \boldsymbol{Z}_{2}$ and that there are two nontrivial fixed point subspaces,

$$
\Sigma_{ \pm}=\{(A, B) \mid A= \pm B\},
$$

corresponding to $\operatorname{Fix}\left(\kappa_{0}\right)$ and $\operatorname{Fix}\left(\kappa_{0} R\right)$, respectively, and representing in-phase (synchronous) and out-of-phase (antisynchronous) motion. Within each subspace the system behaves as a single oscillator and the coupled linear problem separates into corresponding 2-by-2 blocks. Primary Hopf bifurcations cannot occur.

Within the subspaces $\Sigma_{ \pm}$the real and imaginary parts of the coupling coefficient $\mu$ contribute to damping and detuning, 
respectively. Primary bifurcations in $\Sigma_{ \pm}$therefore occur along curves that are shifted with respect to Eq. (5). Pitchfork bifurcations occur on

$$
f^{2}=\left(1 \mp \mu_{r}\right)^{2}+\left(v \pm \mu_{i}\right)^{2},
$$

while saddle-node bifurcations appear when

$$
f^{2}=\frac{\left[\left(1 \mp \mu_{r}\right)+a\left(v \pm \mu_{i}\right)\right]^{2}}{1+a^{2}} ; \quad v>\left(1 \mp \mu_{r}\right) a \mp \mu_{i}
$$

These bifurcations produce $\boldsymbol{Z}_{2}$-symmetric steady-state solutions of Eqs. (25) denoted by $S_{ \pm}$.

In the case of $a<0$, secondary Hopf bifurcations within the $\Sigma_{ \pm}$subspaces occur when a critical amplitude, depending only on $a$ and $\mu_{r}$, is reached,

$$
|A|^{2}=|B|^{2}=\frac{1 \pm \mu_{r}}{2 a} .
$$

These secondary Hopf instabilities are located on the curves

$$
\begin{aligned}
f^{2} & =\left(\frac{1 \mp \mu_{r}}{2}\right)^{2}+\left[\frac{1 \mp \mu_{r}+2 a\left(v \pm \mu_{i}\right)}{2 a}\right]^{2} ; \\
v & <\left(1 \mp \mu_{r}\right) \frac{a^{2}-1}{2 a} \mp \mu_{i},
\end{aligned}
$$

which terminate at the Bogdanov-Takens points

$$
f=\frac{1 \mp \mu_{r}}{2} \sqrt{1+a^{2}} ; \quad v=\left(1 \mp \mu_{r}\right) \frac{a^{2}-1}{2 a} \mp \mu_{i} .
$$

The Hopf frequency is given by

$$
\omega_{H}=\sqrt{\frac{2\left(1 \mp \mu_{r}\right)}{a}\left[v \pm \mu_{i}+\left(1 \mp \mu_{r}\right) \frac{1-a^{2}}{2 a}\right]} .
$$

Note that taking $\mu \rightarrow-\mu$ switches the roles of the in-phase and out-of-phase solutions, as indicated in Eq. (24).

Nonsymmetric solutions not contained in $\Sigma_{ \pm}$can arise in saddle-node bifurcations or in secondary pitchfork bifurcations. These solutions, with $A \neq \pm B$, are neither synchronous nor antisynchronous and are referred to here as mixed modes $(M)$. They do not arise in primary bifurcations.

Figure 5 shows the bifurcation sets for conservative nonlinearity, $a=0$, and a representative value of the coupling, $\mu=0.05+0.2 i$. There are two pitchfork bifurcations within the invariant subspaces $\Sigma_{ \pm}$(labeled $\mathrm{P}_{ \pm}$) defined by Eq. (26) and, in the subcritical regime, associated saddle-node bifurcations (labeled $\mathrm{SN}_{ \pm}$). In addition to these single oscillator instabilities, there is a smooth curve of symmetry-breaking (pitchfork) bifurcations from the $\Sigma_{-}$subspace to mixed mode (asymmetric) states and two more symmetry-breaking bifurcations, one from $\Sigma_{+}$and one from $\Sigma_{-}$emerging from the mode interaction point where $\mathrm{P}_{ \pm}$cross; these three dash-dotted curves are labeled $\mathrm{SB}_{ \pm}$according to the subspace of the (pure) mode undergoing the instability. Finally, near the mode interaction point is a cusp that forms the leftmost boundary of two saddle-node bifurcations on the mixed mode branch.

The bifurcation diagram in Fig. 6 shows the three bifurcations that occur for $v=-1.5$. There is a primary pitchfork bifurcation in $\Sigma_{+}$at $f=1.61$ and in $\Sigma_{-}$at $f=$ 1.998; these produce the two $Z_{2}$-symmetric branches, $S_{ \pm}$. A symmetry-breaking bifurcation on the $S_{-}$branch at $f=2.196$ stabilizes that branch and gives rise to a branch of (four symmetry-related) asymmetric $M$ states.

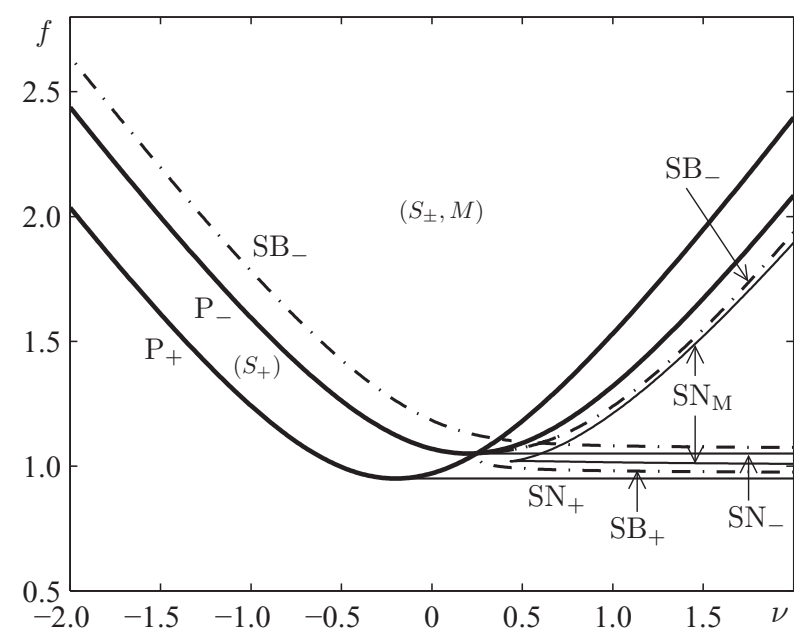

FIG. 5. Bifurcation sets for $\phi=0, a=0, \mu=0.05+0.2 i$ : primary pitchfork in $\Sigma_{ \pm}$subspaces ( $\mathrm{P}_{ \pm}$, thick solid curves), saddle-node in $\Sigma_{ \pm}\left(\mathrm{SN}_{ \pm}\right.$, thin solid lines), symmetry-breaking (pitchfork) from $\Sigma_{ \pm}$to mixed mode state ( $\mathrm{SB}_{ \pm}$, dash-dotted curves), saddle-node on mixed mode state $\left(\mathrm{SN}_{\mathrm{M}}\right.$, thin solid curves). In the larger regions the types of solutions that exist there are indicated in parentheses.

Phase portraits illustrating the different types of solutions are shown in Fig. 7 for the parameters of Fig. 6 and $f=$ 2.5; here $A=A_{r}+i A_{i}$ and $B=B_{r}+i B_{i}$. The projections onto $\left(A_{r}, B_{r}\right)$ and $\left(A_{i}, B_{i}\right)$ show the invariant subspaces $\Sigma_{ \pm}$ where the solutions $S_{ \pm}$are found, and the effect of the symmetry-breaking bifurcation from $S_{-}$that produces the (four) asymmetric $M$ states.

With positive detuning the scenario is more complex, as illustrated for $v=1.5$ in Fig. 8 . The primary pitchfork

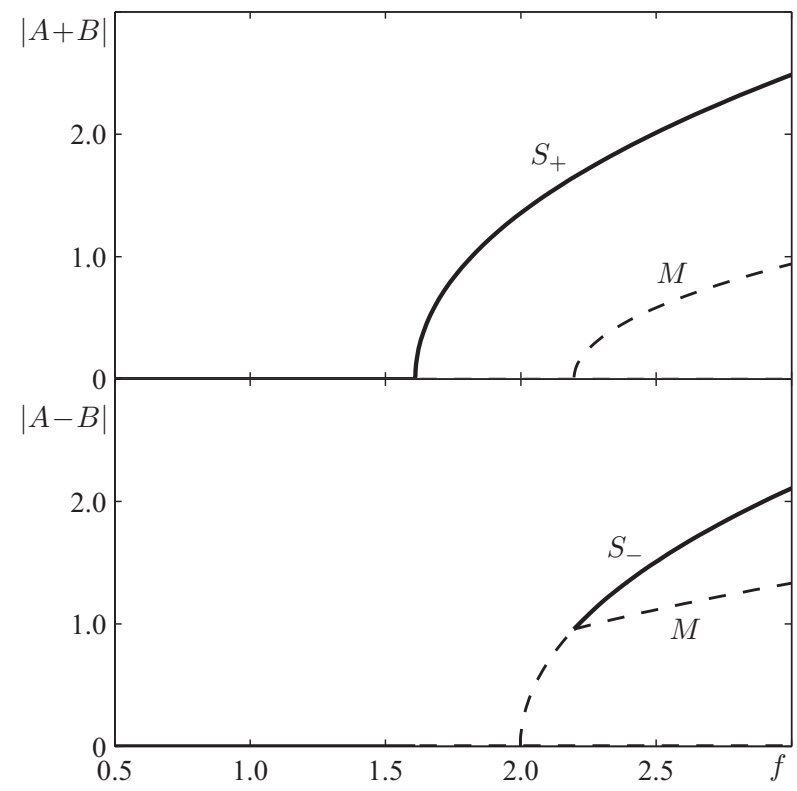

FIG. 6. Bifurcation diagram for $v=-1.5, \phi=0, a=0, \mu=$ $0.05+0.2 i$ showing $|A+B|$ (projection onto $\Sigma_{+}$) and $|A-B|$ (projection onto $\Sigma_{-}$). Solid (dashed) curves denote stable (unstable) solutions. Solutions in the $\Sigma_{ \pm}$subspaces are labeled with $S_{ \pm}$and asymmetric mixed modes with $M$. 
(a)
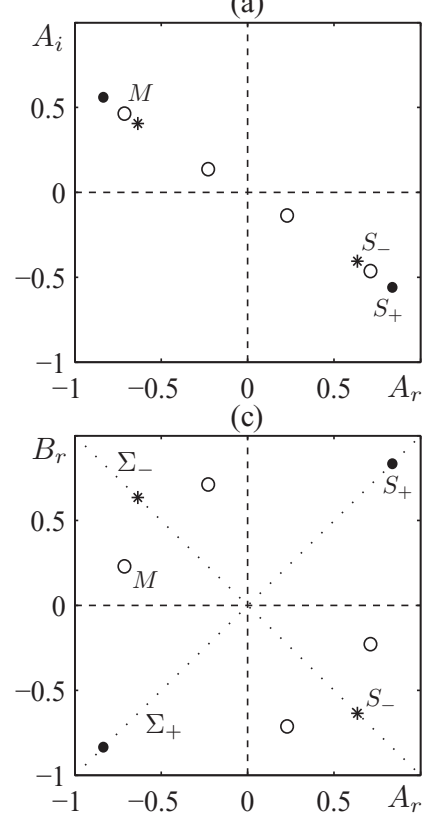

(b)

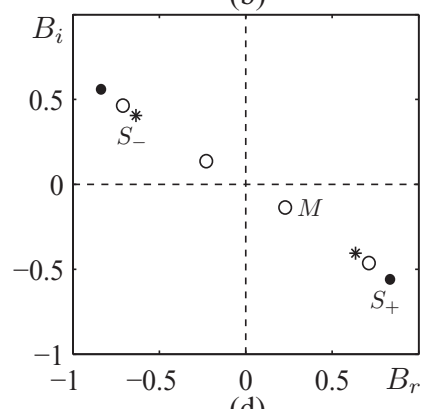

(d)

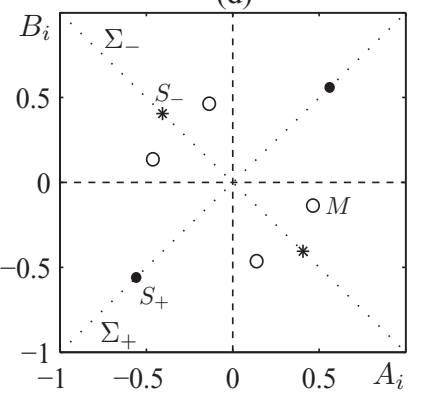

FIG. 7. Phase space portraits corresponding to the case of Fig. 6 with $f=2.5$. There are two stable steady states $S_{+}$(solid dots) in $\Sigma_{+}$, two stable steady states $S_{-}$(asterisks) in $\Sigma_{-}$and four unstable asymmetric steady states $M$ (open circles); one representative solution on each group orbit is labeled. Panels (a) and (b) show projections onto $A=A_{r}+i A_{i}$ and $B=B_{r}+i B_{i}$, respectively, while the projections in (c) and (d) illustrate the interchange symmetry (21). The trivial state is not shown.

bifurcations in $\Sigma_{ \pm}$, at $f=1.947$ and $f=1.671$, respectively, are subcritical. The nontrivial $\boldsymbol{Z}_{2}$-symmetric branches created at these bifurcations, $S_{ \pm}$, are stabilized (within the corresponding subspace) in saddle-node bifurcations at $f=0.95$ and $f=1.05$, respectively. In the intermediate region between these saddle-node bifurcations and the original pitchfork bifurcations is a branch of asymmetric $M$ states connecting to $S_{+}$at $f=0.977$ and $S_{-}$at $f=1.548$. This $M$ branch undergoes two saddle-node bifurcations, at $f=1.011$ and $f=1.52$; between these values are three distinct $M$ solutions, one of which is stable. A separate unstable asymmetric $M$ branch bifurcates at $f=1.077$ from $S_{-}$and stabilizes it. Both $S_{ \pm}$branches are thus stable for large forcing values.

We remark that the case $a=\mu_{r}=0$, where neither nonlinearity nor coupling contribute to dissipation, is degenerate in certain respects. The three leftmost saddle-node bifurcations, for example, on the $S_{ \pm}$and $M$ branches, occur exactly at $f=1$, while the two symmetry-breaking bifurcations nearest $f=1$, one on $S_{+}$and one on $S_{-}$, coincide as well.

It is also worth pointing out that, in the limit of large forcing, there are always four stable steady states and four unstable steady states. This fact, which can be seen in Figs. 6 and 8 and in the following two sections, is independent of the forcing phase $\phi$ because, at large amplitude, the coupling term becomes negligible compared to the nonlinearity. In this limit the coupled system will increasingly resemble two uncoupled oscillators. The uncoupled system has four stable states, corresponding to the four possible phases with both

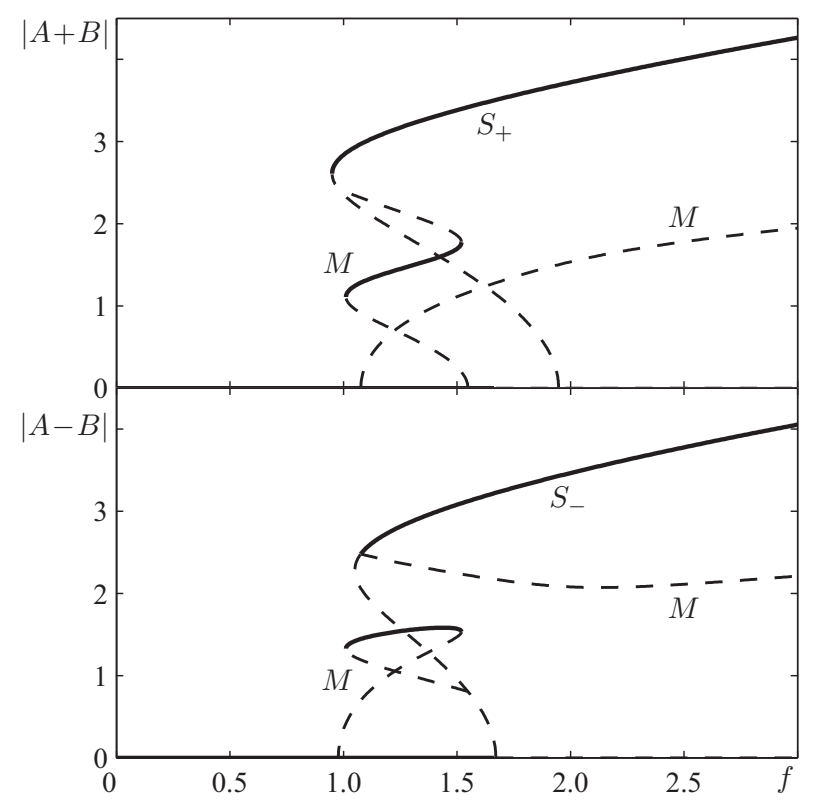

FIG. 8. Bifurcation diagram for $v=1.5, \phi=0, a=0, \mu=$ $0.05+0.2 i$ showing $|A+B|$ and $|A-B|$. Solid (dashed) curves denote stable (unstable) solutions. Solutions in the $\Sigma_{ \pm}$subspaces are labeled with $S_{ \pm}$and asymmetric mixed modes with $M$.

oscillators excited, and four unstable states, corresponding to the four possible equilibria with only one oscillator excited. Thus, one does not expect complicated dynamics for large forcing regardless of the forcing phase; the residual symmetry that depends on $\phi$ is important near onset where the weak coupling is relevant, but not at large amplitude.

\section{Antisymmetric forcing, $\phi=\pi$}

With antisymmetric forcing the governing equations for the two coupled oscillators are

$$
\begin{aligned}
& \dot{A}=(-1+i v) A+f \bar{A}-(a+i)|A|^{2} A+\mu B \\
& \dot{B}=(-1+i v) B-f \bar{B}-(a+i)|B|^{2} B+\mu A
\end{aligned}
$$

Recall that these equations are equivariant under the cyclic permutation $\rho:(A, B) \rightarrow i(B, A)$, which generates a representation of the group $C_{4}$. There are no nontrivial fixed point subspaces and, consequently, the primary bifurcation cannot be reduced to the case of a single oscillator. The four eigenvalues describing the stability of the trivial state are given by

$$
\begin{aligned}
& \lambda_{ \pm}=-1 \pm i \mu_{i}+\sqrt{f^{2}-v^{2}+\mu_{r}^{2} \pm 2 i v \mu_{r}}, \\
& \beta_{ \pm}=-1 \pm i \mu_{i}-\sqrt{f^{2}-v^{2}+\mu_{r}^{2} \pm 2 i v \mu_{r}}
\end{aligned}
$$

which are, in general, complex.

A Hopf bifurcation occurs along the curve

$$
f^{2}=\left(1+v^{2}\right)\left(1-\mu_{r}^{2}\right) ; \quad v \neq-\frac{\mu_{i}}{\mu_{r}},
$$


where $\lambda_{ \pm}= \pm i \Omega$, creating a $\boldsymbol{C}_{4}$-symmetric periodic orbit $\left(P_{\boldsymbol{C}_{4}}\right)$. The Hopf frequency is given by

$$
\Omega=\mu_{i}+v \mu_{r}
$$

We emphasize that this is a primary bifurcation, in contrast to the secondary Hopf bifurcations that already arise in a single oscillator system with $a<0$ [see Eq. (9) and Eq. (29)]. It results from the linear coupling and the symmetry properties of antisymmetric forcing and not nonlinear effects. Note also that the Hopf frequency $\Omega$ is small if the coupling $\mu$ is small, as assumed in this analysis.

If the interaction is conservative $\left(\mu_{r}=0\right)$, then the location of the primary instability, which is now oscillatory rather than steady, is unchanged from the uncoupled problem and given by $f^{2}=1+v^{2}$. If the interaction is not conservative, then the Hopf bifurcation is shifted to lower forcing values; i.e., the interaction is always destabilizing.

The periodic modulated solution $P_{C_{4}}$ generated in the primary Hopf bifurcation is not expected to persist for large values of $f$, where the influence of the coupling term diminishes compared to the nonlinear and forcing terms, but only an $O(|\mu|)$ distance above onset. Beyond that point, this $\boldsymbol{C}_{4}$-symmetric limit cycle must be replaced by steady states $(S)$ approximating the solutions of the uncoupled problem given by Eq. (7). We find that this transition generally takes the form of a saddle-node heteroclinic bifurcation. This heteroclinic bifurcation is codimension one because the four steady states involved lie on the same group orbit.

The bifurcation sets corresponding to the conservative case $a=\mu_{r}=0$ are shown in Fig. 9. The primary Hopf $(\mathrm{H})$ bifurcation is supercritical for $v<0$ and subcritical for $v>0$, just as the primary symmetry-breaking bifurcation is for a single oscillator (cf. Fig. 1). In the supercritical region, the $\boldsymbol{C}_{4}$-symmetric periodic orbit created in the primary bifurcation is stable and exists until a saddle-node heteroclinic (SN Het) bifurcation. In the subcritical region for $v \leqslant 1.322$, this stable periodic orbit exists between the saddle-node heteroclinic bifurcation and a saddle-node of periodic orbits (SN Per); the unstable $\boldsymbol{C}_{4}$-symmetric periodic orbit involved in this saddle-node is the one generated in the subcritical primary Hopf bifurcation. For $v \geqslant 1.322$ there is a curve of saddle-node (SN) bifurcations generating (four) asymmetric steady states and, for $v \geqslant 1.466$, a second such curve originating in a cusp. Bogdanov-Takens (BT) points on each of these SN curves form the boundaries of associated Hopf and homoclinic (Hom) bifurcation sets (only visible in the lower plot) between which (four) asymmetric periodic solutions can be found. The termination of these two bifurcation sets at the BT point on the right is preceded by a couple of transitions. First, the homoclinic bifurcation set collides with the upper SN curve, marking the upper boundary of a set of saddle-node homoclinic (SN Hom) bifurcations. The homoclinic bifurcation then detaches again from the SN curve before reaching the cusp and moves toward the Bogdanov-Takens point on the lower SN curve.

As with the case of symmetric forcing, the bifurcation structure is quite complex for positive detuning and is characterized for $\phi=\pi$ by multiple saddle-node bifurcations, secondary Hopf bifurcations, and Bogdanov-Takens points. The periodic solutions characteristic of antisymmetric forcing (a)
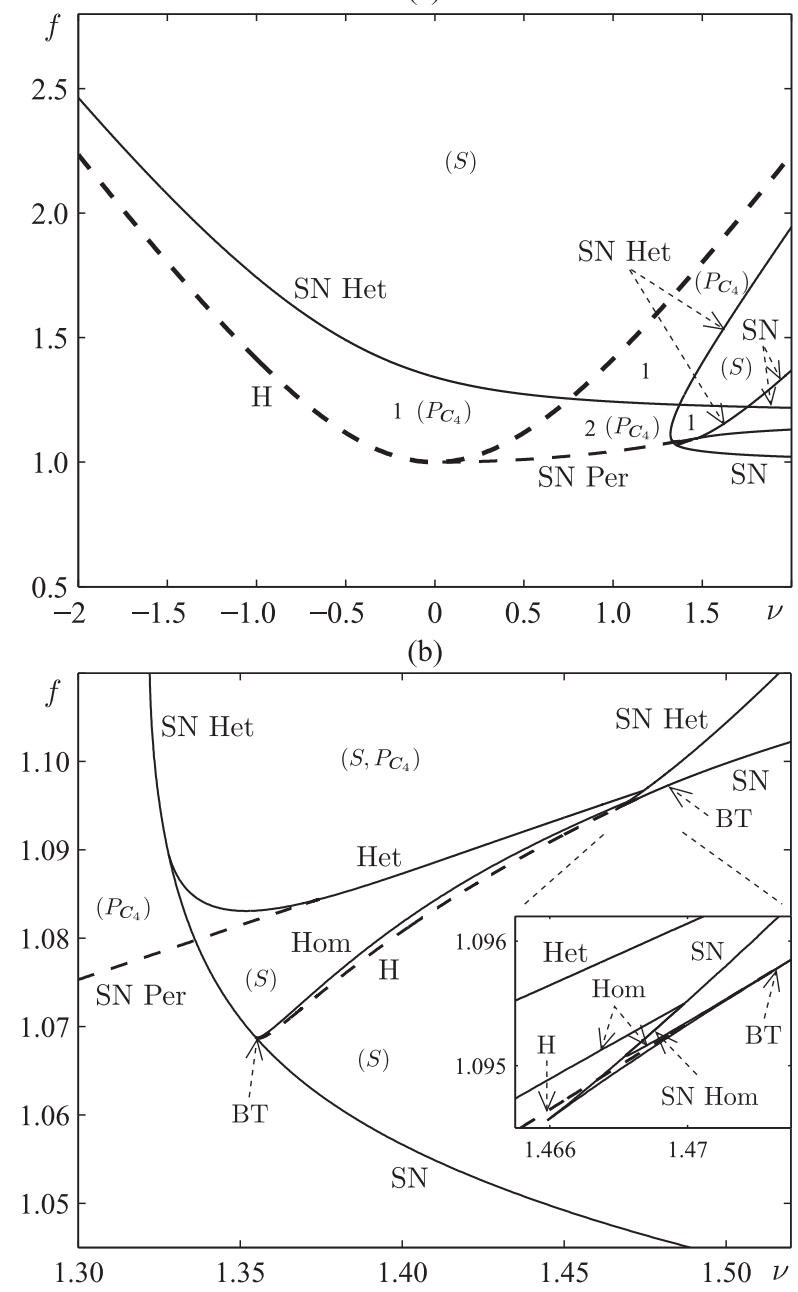

FIG. 9. Bifurcation sets for $\phi=\pi, a=0, \mu=0.2 i$ : Hopf ( $\mathrm{H}$, dashed curves) and saddle-node of periodic orbits (SN Per, dashed curve), saddle-node ( $\mathrm{SN}$ ), heteroclinic (Het), saddle-node heteroclinic (SN Het), homoclinic (Hom), saddle-node homoclinic (SN Hom), Bogdanov-Takens points (BT). In the larger regions, the types of solutions that exist there are indicated in parentheses. In (a) the regions of one and two $\boldsymbol{C}_{4}$-symmetric periodic orbits $\left(P_{\boldsymbol{C}_{4}}\right)$ are further marked with " 1 " and " 2 ", respectively. In (b) a close-up of the complex region between two BT points near the cusp of SN bifurcations is shown, as is, in the inset, a further magnification showing the cusp region.

exist in a finite region near the primary Hopf bifurcation and are destroyed in global bifurcations (heteroclinic or saddle-node heteroclinic).

Several bifurcation diagrams are shown in Fig. 10. For $v=-0.5$ and $v=0.5$, the $\boldsymbol{C}_{4}$-symmetric periodic orbit $\left(P_{\boldsymbol{C}_{4}}\right)$ created in the primary bifurcation at $f=1.118$ terminates in a saddle-node heteroclinic bifurcation at $f=1.491$ and $f=1.273$, respectively; for $v=0.5$, the periodic orbit first undergoes a saddle-node bifurcation at $f=1.011$. The stable and unstable branches of steady states $(S)$ created in the saddlenode heteroclinic bifurcation persist for large $f$.

For $v=1.4$, this scenario is interrupted by an isola of steady states existing for $1.057 \leqslant f \leqslant 1.271$. The initial (unstable) $\boldsymbol{C}_{4}$-symmetric periodic orbit created via Hopf bifurcation 


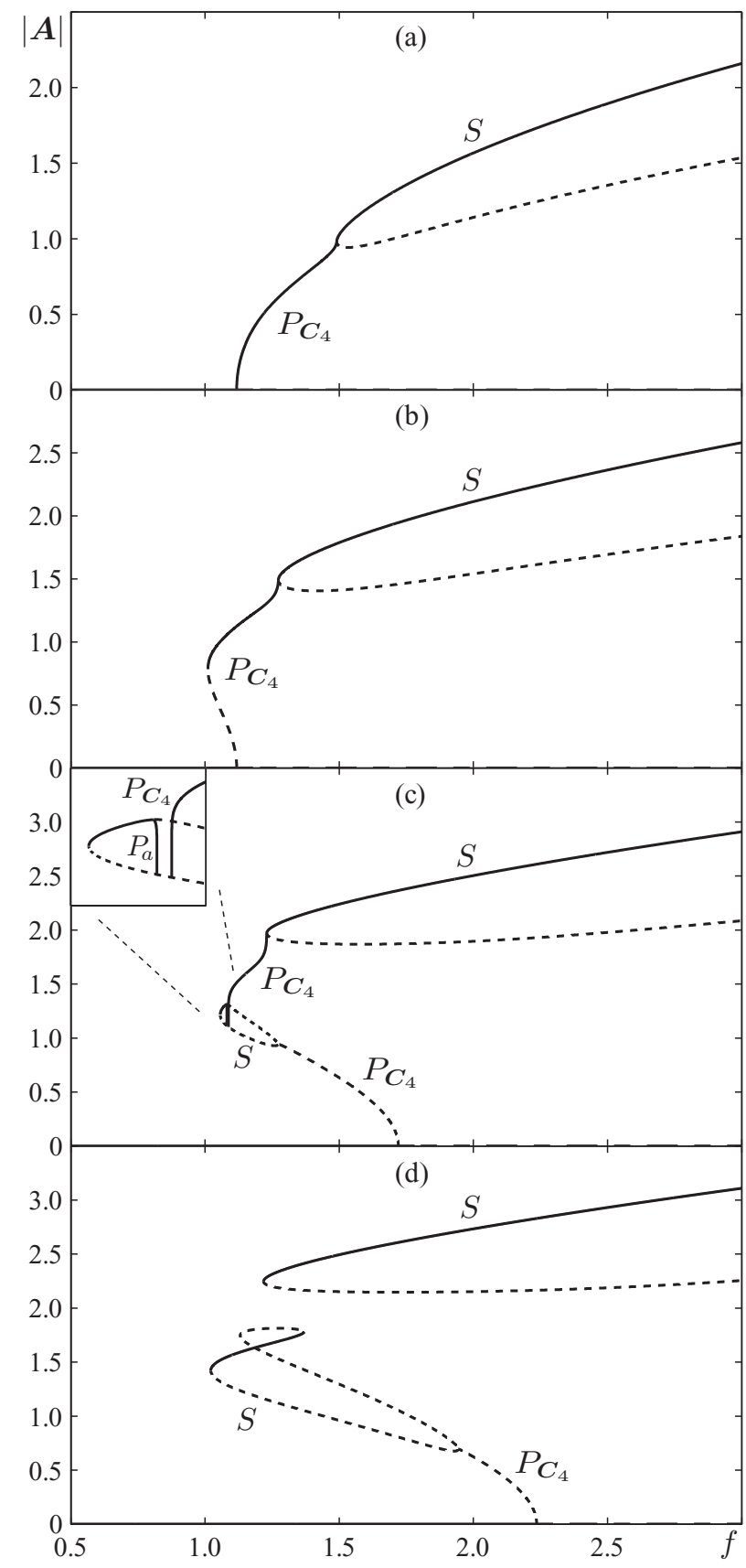

FIG. 10. Bifurcation diagrams for $\phi=\pi, a=0, \mu=0.2 i$ showing the norm $|\boldsymbol{A}|=|(A, B)|$ of steady states $(S), \boldsymbol{C}_{4}$-symmetric periodic orbits $\left(P_{C_{4}}\right)$, and asymmetric periodic orbits $\left(P_{a}\right)$ : (a) $v=$ -0.5 , (b) $v=0.5$, (c) $v=1.4$, (d) $v=2$. For $v=1.4$, there is a secondary supercritical Hopf bifurcation on the isola of steady states that generates stable asymmetric periodic orbits, shown in the inset. Stable (unstable) solutions are shown with solid (dashed) lines.

at $f=1.721$ is destroyed in a saddle-node heteroclinic bifurcation at the right boundary of this isola. A heteroclinic bifurcation on the lower branch of the isola generates a stable $\boldsymbol{C}_{4}$-symmetric periodic orbit, which terminates on the unbounded steady state branch in a saddle-node heteroclinic bifurcation at $f=1.23$. A secondary Hopf bifurcation at $f=1.0808$ on the upper branch of the isola produces stable (a)

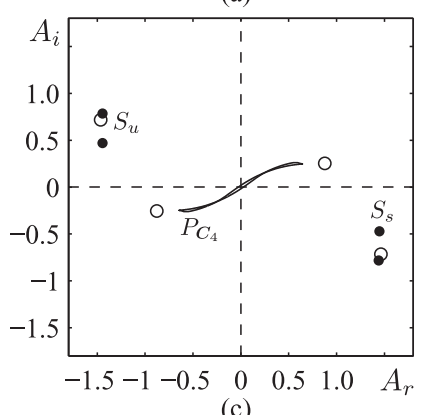
(c)

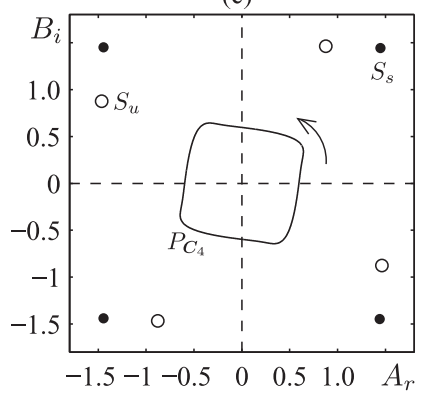

(b)

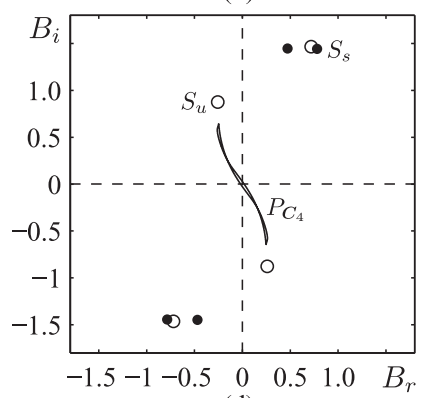

(d)

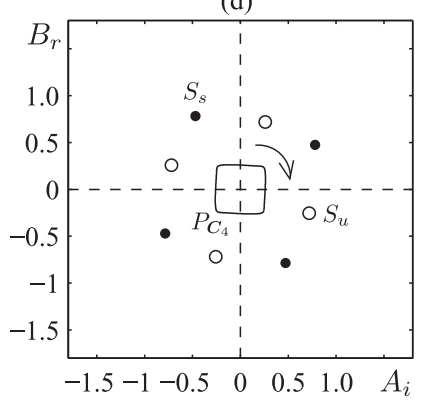

FIG. 11. Phase space portraits corresponding to the case of Fig. 10 (c) with $f=1.5$. There are four stable steady states $S_{s}$ (solid dots) and four unstable steady states $S_{u}$ (open circles) related by the symmetry (22); one representative solution on each group orbit is labeled. The $\boldsymbol{C}_{4}$-symmetric periodic orbit, $P_{C_{4}}$, is also shown. Panels (a) and (b) show projections onto $A=A_{r}+i A_{i}$ and $B=B_{r}+i B_{i}$, respectively, while the projections in (c) and (d) illustrate the $\boldsymbol{C}_{4}$ symmetry. The trivial state is not shown.

asymmetric periodic orbits $\left(P_{a}\right)$ that quickly collide with the lower branch in a homoclinic bifurcation at $f=1.0818$.

With $v=2$, the primary subcritical Hopf bifurcation occurs at $f=2.236$ and the $\boldsymbol{C}_{4}$-symmetric periodic orbit created there again terminates in a saddle-node heteroclinic bifurcation, at $f=1.944$. The lower branch of steady states created here is stabilized in a second saddle-node bifurcation at $f=1.021$ and destabilized again in another saddle-node at $f=1.368$, then once more at $f=1.131$. The unbounded steady states appear in a saddle-node bifurcation at $f=1.218$.

Phase portraits illustrating both steady and periodic solutions are shown in Fig. 11 for the parameters of Fig. 10(c) and $f=1.5$. At this forcing value there are four stable steady states, related by the symmetry $\rho$ of Eq. (22), and four unstable steady states, likewise related by the rotation $\rho$. The projections onto $\left(A_{r}, B_{i}\right)$ and $\left(A_{i}, B_{r}\right)$ best reveal this $\boldsymbol{C}_{4}$ symmetry and further show that the limit cycle itself is symmetric.

In the case of real coupling $\mu$ there are differences with the scenario described above, most notably the absence of Bogdanov-Takens points in the cusp region [cf. Fig. 9(b)]. Because of degeneracies that make the numerical calculations difficult in the purely real case, we show the bifurcation sets for $\mu=0.2+0.04 i$ in Fig. 12. With complex coupling there is a double-zero (degenerate Hopf) bifurcation at $v=-\mu_{i} / \mu_{r}$, where the Hopf frequency $\Omega$ vanishes; see Eq. (35). Note that we use the term "double-zero bifurcation" to refer to the case of a pair of zero eigenvalues described by a semisimple (diagonalizable) Jordan block, while the term 


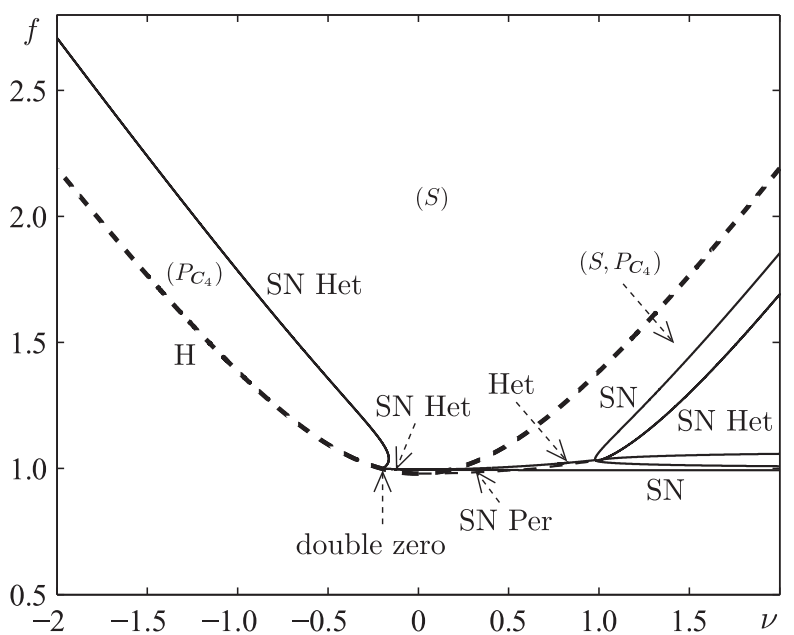

FIG. 12. Bifurcation sets for $\phi=\pi, a=0, \mu=0.2+0.04 i$ : Hopf $(\mathrm{H}$, dashed curve) and saddle-node of periodic orbits ( $\mathrm{SN}$ Per, dashed curve), saddle-node (SN), heteroclinic (Het), saddle-node heteroclinic (SN Het), and double-zero point. In the larger regions, the types of solutions that exist there are indicated in parentheses.

"Bogdanov-Takens bifurcation" refers throughout this paper to the usual nonsemisimple case; some authors use these terms synonymously [29]. In this case, since the codimension-two point corresponds to the vanishing of the Hopf frequency along a curve of Hopf bifurcations, it may also be called a degenerate Hopf bifurcation.

The curve of saddle-node heteroclinic bifurcations that follows the primary Hopf bifurcation for negative $v$ appears to pinch off here at the double-zero point $(v=-0.2$ for this choice of coupling), then continues to the right before separating into distinct saddle-node and heteroclinic bifurcations at about $v=-0.132$; this region is shown in more detail in Fig. 13(a). We note that the details of this double-zero point can be very complicated [38] and include, in a small neighborhood, many other bifurcations: pitchfork, homoclinic, heteroclinic, gluing, Bogdanov-Takens, etc. We did not attempt to resolve these in Fig. 12 or Fig. 13.

The curve of heteroclinic bifurcations collides with the (smooth) curve of saddle-node bifurcations at $v=0.98$, creating a short region of saddle-node heteroclinic bifurcations, before detaching again at $\mu=0.982$ and heading toward the cusp on the other curve of saddle-nodes and merging (just beyond the cusp) with the upper branch. Figure 13(b) shows this scenario in more detail. Note the absence, compared to Fig. 9, of Bogdanov-Takens points and the associated asymmetric periodic states.

The bifurcation sets in the examples above were obtained for $a=0$. In this case the transition from supercritical to subcritical Hopf occurs at $v=0$ and the complicated (cusp) region with multiple saddle-node bifurcations appears for $v \gtrsim 1$. If $a \neq 0$ these features can be shifted left $(a<0)$ or right $(a>0)$. Figure 14 shows the transition from supercritical to subcritical Hopf bifurcation in the $(v, a)$ plane, which does not depend on $\mu_{i}$. If $\mu_{r}=0$, the transition occurs along the line $a=v$, while for $\mu_{r}>0$ there is a critical value of $|a|=a_{c}$ such that for $a>a_{c}$ the Hopf bifurcation is supercritical for (a)

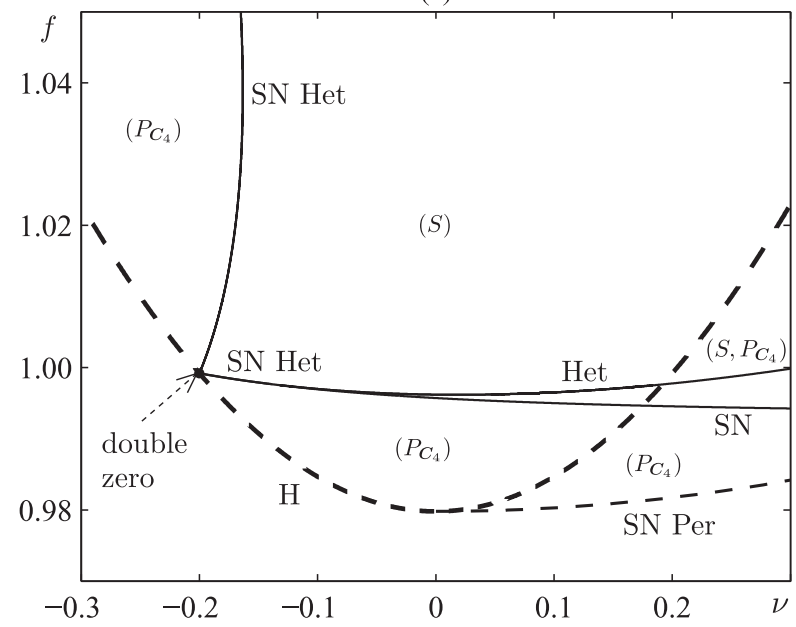

(b)

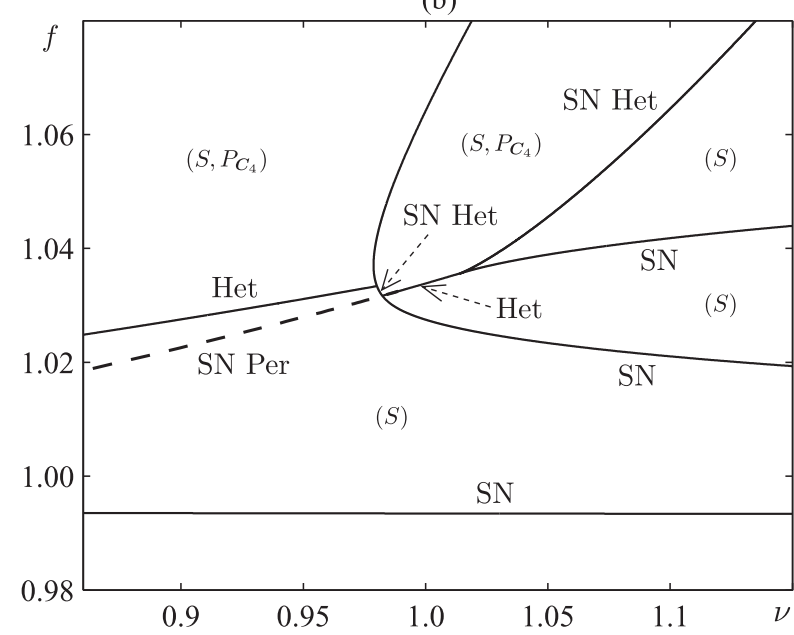

FIG. 13. (a) Close-up of the double-zero bifurcation point and (b) cusp region of Fig. 12 showing bifurcation sets: Hopf $(\mathrm{H}$, dashed curve), saddle-node of periodic orbits (SN Per, dashed curve), saddlenode (SN), saddle-node heteroclinic (SN Het), and heteroclinic (Het). The heteroclinic bifurcation set near the cusp does not collide there, but with the upper branch, although this cannot be distinguished on the scale of the plot. In the larger regions, the types of solutions that exist there are indicated in parentheses.

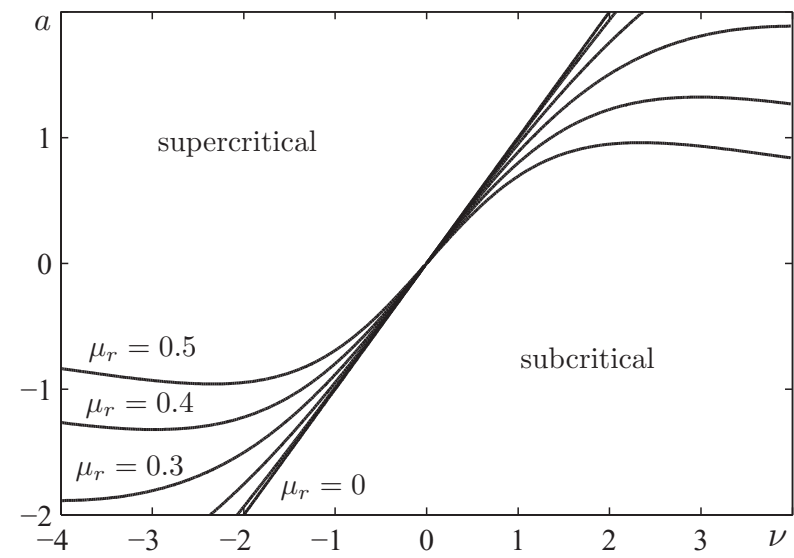

FIG. 14. Contours showing the transition from supercritical to subcritical Hopf bifurcation for coupling values with $\mu_{r}=$ $0,0.1,0.2,0.3,0.4,0.5$. The transition is independent of $\mu_{i}$. 
all $v$ and for $a<-a_{c}$ the Hopf bifurcation is subcritical for all $v$. For $\mu_{r}=0.4$, for example, this critical value is $a_{c} \sim 2.98$.

\section{General forcing phase}

With $\phi \neq 0, \pi$ the governing equations (16) do not simplify. They possess only the subharmonic symmetry $R$ of Eq. (20). This case is intermediate between symmetric forcing $(\phi=0)$, where the primary instabilities can be identified with the pitchfork bifurcations of single oscillators, and antisymmetric forcing $(\phi=\pi)$, where the primary instability is a Hopf bifurcation. Transitions between steady and oscillatory behavior are observed as parameters are varied.

In the limiting case of conservative $\left(\mu_{r}=0\right)$ and purely dissipative coupling $\left(\mu_{i}=0\right)$ the location of the primary bifurcations are given by relatively simple expressions.

In the case of conservative coupling, when $\mu_{r}=0$, a primary Hopf bifurcation, which produces $\boldsymbol{Z}_{2}$-symmetric periodic orbits $\left(P_{Z_{2}}\right)$, occurs for

$$
|v|<\sqrt{1+\mu_{i}^{2}} \tan \frac{\phi}{2}
$$

along the curve

$$
f^{2}=\frac{\left(1+\mu_{i}^{2}\right)\left(1+v^{2}\right)}{1+\mu_{i}^{2} \sin ^{2} \frac{\phi}{2}},
$$

and the Hopf frequency satisfies

$$
\Omega=\mu_{i} \sqrt{\frac{\left(1+\mu_{i}^{2}\right) \sin ^{2} \frac{\phi}{2}-v^{2} \cos ^{2} \frac{\phi}{2}}{1+\mu_{i}^{2} \sin ^{2} \frac{\phi}{2}}} .
$$

Primary pitchfork bifurcations, producing steady states $(S)$, occur for

$$
|v| \geqslant \tan \frac{\phi}{2} \sqrt{1+\mu_{i}^{2} \cos ^{2} \frac{\phi}{2}}
$$

on the curves

$$
\begin{aligned}
f^{2}=1 & +v^{2}+\mu_{i}^{2} \cos \phi \\
& \pm 2 \sqrt{v^{2} \mu_{i}^{2} \cos ^{2} \frac{\phi}{2}-\mu_{i}^{2} \sin ^{2} \frac{\phi}{2}-\frac{\mu_{i}^{4}}{4} \sin ^{2} \phi .}
\end{aligned}
$$

The codimension-two points where the pitchfork and Hopf bifurcation curves meet are given by

$$
(\nu, f)=\sqrt{1+\mu_{i}^{2}}\left( \pm \tan \frac{\phi}{2}, \sec \frac{\phi}{2}\right) .
$$

Such transitions are referred to here as Bogdanov-Takens bifurcations with $\boldsymbol{Z}_{2}$ symmetry (see, e.g., [38,39]) since the pair of zero eigenvalues corresponds to a nonsemisimple block of the Jordan normal form. Note that a Bogdanov-Takens bifurcation with $\boldsymbol{Z}_{2}$ symmetry will occur when the equilibrium in question is the symmetric (trivial) state $A=B=0$, while Bogdanov-Takens bifurcations occurring on finite-amplitude branches (where the $\boldsymbol{Z}_{2}$ symmetry has already been broken) will be the usual (asymmetric) kind.

In the case of purely dissipative coupling, with $\mu_{i}=0$, primary Hopf bifurcation occurs for

$$
|v|>\sqrt{1-\mu_{r}^{2}} \cot \frac{\phi}{2}
$$

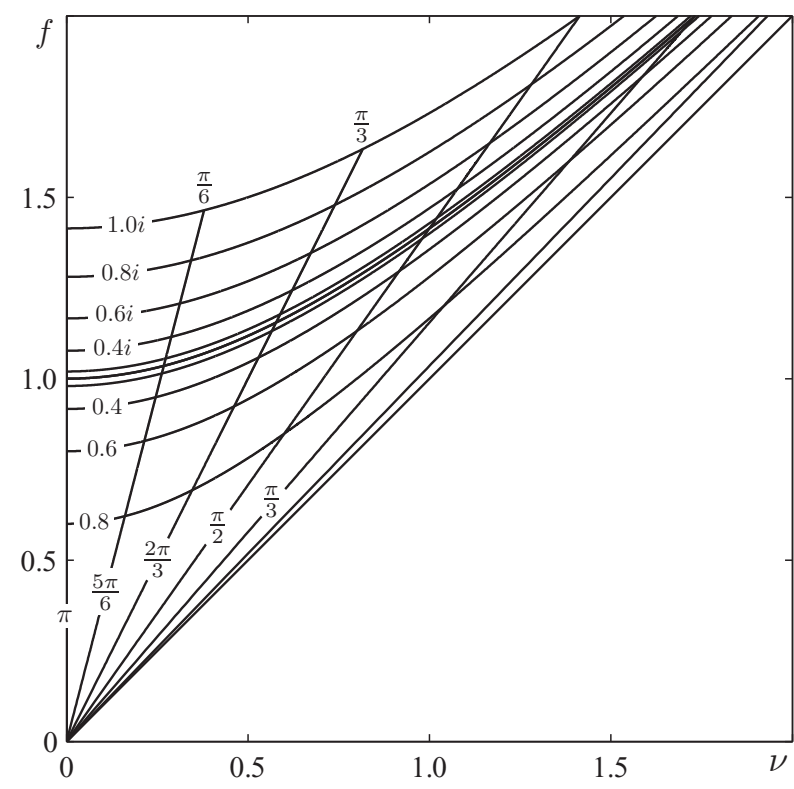

FIG. 15. Contour map showing the location of the $\boldsymbol{Z}_{2}$-symmetric Bogdanov-Takens point with $v>0$ in the conservative and purely dissipative cases, as given by Eqs. (41) and (47) . Curves for $v<$ 0 are obtained by reflection. Curves are shown for constant $\mu$, in steps of $0.2 i(\mu \in \operatorname{Im})$ and $0.2(\mu \in \mathrm{Re})$, and for constant $\phi$ (radial lines), in steps of $\pi / 6$. The central curve intersecting $(\nu, f)=(0,1)$ corresponds to the uncoupled case $\mu=0$.

along the curve

$$
f^{2}=\frac{\left(1-\mu_{r}^{2}\right)\left(1+v^{2}\right)}{1-\mu_{r}^{2} \cos ^{2} \frac{\phi}{2}},
$$

and the Hopf frequency is

$$
\Omega=\mu_{r} \sqrt{\frac{\left(\mu_{r}^{2}-1\right) \cos ^{2} \frac{\phi}{2}+v^{2} \sin ^{2} \frac{\phi}{2}}{1-\mu_{r}^{2} \cos ^{2} \frac{\phi}{2}} .}
$$

Primary pitchfork bifurcations occur for

$$
|v| \leqslant \cot \frac{\phi}{2} \sqrt{1-\mu_{r}^{2} \sin ^{2} \frac{\phi}{2}}
$$

on the curves

$$
\begin{aligned}
f^{2} & =1+v^{2}+\mu_{r}^{2} \cos \phi \\
& \pm 2 \sqrt{\mu_{r}^{2} \cos ^{2} \frac{\phi}{2}-v^{2} \mu_{r}^{2} \sin ^{2} \frac{\phi}{2}-\frac{\mu_{i}^{4}}{4} \sin ^{2} \phi}
\end{aligned}
$$

Bogdanov-Takens bifurcations with $\boldsymbol{Z}_{2}$ symmetry terminate the set of Hopf bifurcations at

$$
(\nu, f)=\sqrt{1-\mu_{r}^{2}}\left( \pm \cot \frac{\phi}{2}, \csc \frac{\phi}{2}\right) .
$$

Figure 15 shows the location of the $Z_{2}$-symmetric Bogdanov-Takens points in the conservative and purely dissipative cases. Note that for $\mu$ imaginary (upper set of curves in Fig. 15), increasing $\phi$ moves the $Z_{2}$-symmetric Bogdanov-Takens points outward, while for $\mu$ real (lower set of curves) the $\boldsymbol{Z}_{2}$-symmetric Bogdanov-Takens points move 


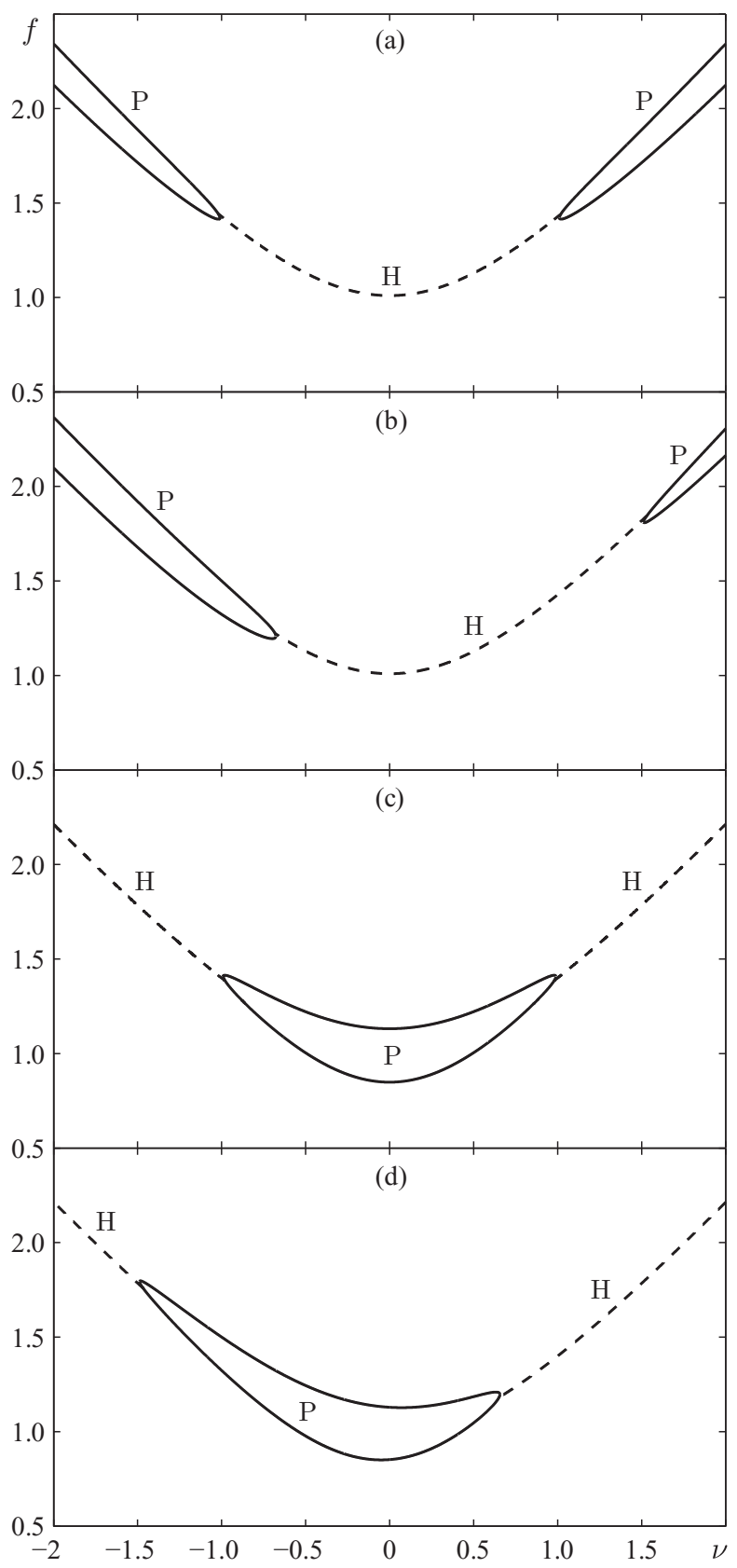

FIG. 16. Primary Hopf $(\mathrm{H})$ and pitchfork $(\mathrm{P})$ bifurcation sets for (a) $\mu=0.2 i$, (b) $\mu=0.04+0.2 i$, (c) $\mu=0.2$, and (d) $\mu=0.2+$ $0.04 i$. $\boldsymbol{Z}_{2}$-symmetric Bogdanov-Takens points (not marked) lie on the intersection.

inward with increasing $\phi$. In both cases the region of Hopf bifurcation increases with $\phi$. Note that, although Eqs. (41) and (47) have well-defined limits as $\mu_{i} \rightarrow 0$ and $\mu_{r} \rightarrow 0$, respectively, there are no primary Hopf bifurcations in the uncoupled problem since the Hopf frequency $\Omega$ is zero; see Eqs. (38) and (44). For $\mu=0$ there is a double-zero (dual pitchfork) bifurcation for any value of detuning.

Figure 16 shows the primary Hopf and pitchfork bifurcation sets corresponding to two sets of values from Fig. 15: $\phi=\pi / 2$, $\mu=0.2 i$, shown in Fig. 16(a), with $\boldsymbol{Z}_{2}$-symmetric BogdanovTakens points at $(\nu, f)=( \pm 1.0198,1.4422)$, and $\phi=\pi / 2$, (a)

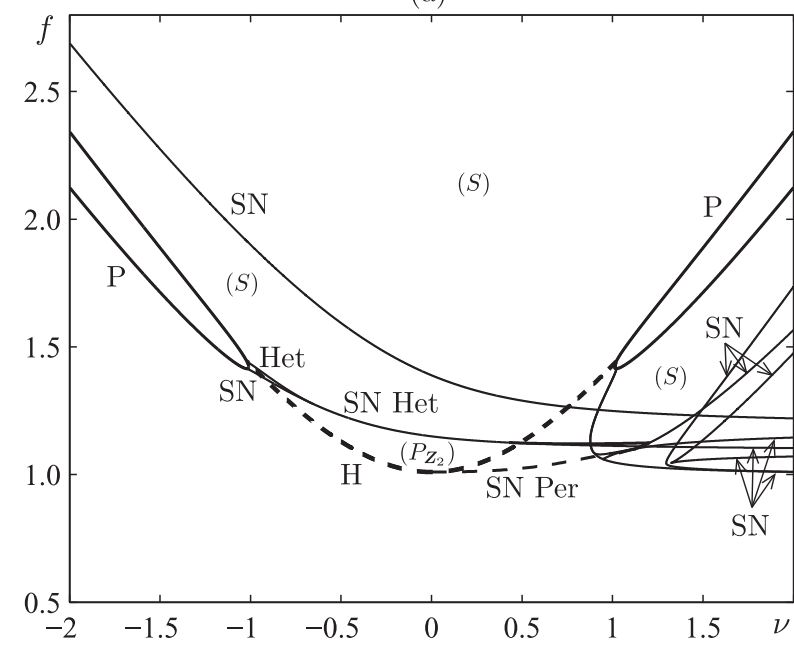

(b)

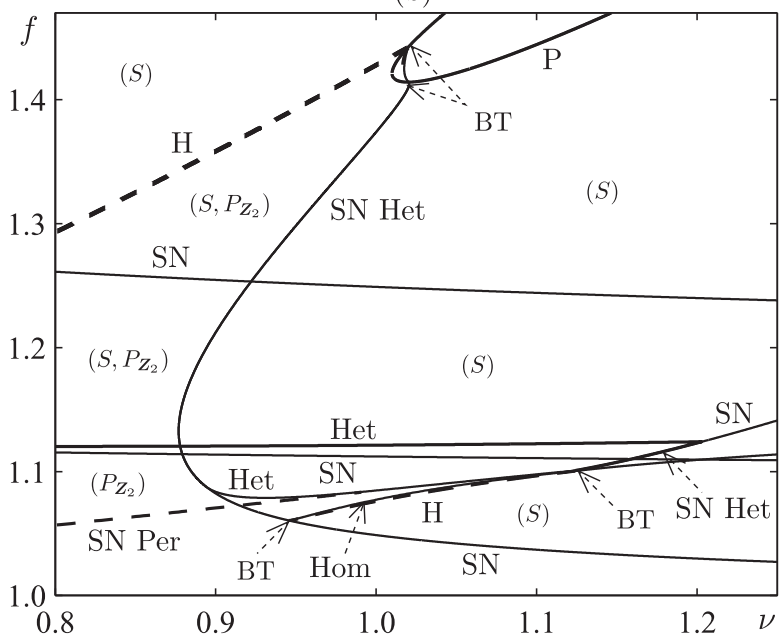

FIG. 17. (a) Bifurcation sets for $\phi=\pi / 2, a=0, \mu=0.2 i$ : Hopf ( $\mathrm{H}$, dashed curves), saddle-node of periodic orbits (SN Per, dashed curve), saddle-node (SN), heteroclinic (Het), saddle-node heteroclinic (SN Het), homoclinic (Hom), Bogdanov-Takens points (BT). Some bifurcation sets (SN Per, homoclinic, and gluing bifurcations, for example) are hard to see on the scale of the plot and are not labeled. Panel (b) shows a close-up of the complex transitions for positive detuning. In the larger regions, the types of solutions that exist there are indicated in parentheses.

$\mu=0.2$, shown in Fig. 16(c), with $\boldsymbol{Z}_{2}$-symmetric BogdanovTakens points at $(v, f)=( \pm 0.9798,1.3856)$. Observe that conservative coupling produces a region of Hopf bifurcations centered at $v=0$ with pairs of pitchfork bifurcations outside of this, in accordance with Eqs. (36) and (39). Purely dissipative coupling produces the opposite, a region of pitchfork bifurcations centered at $v=0$ with Hopf bifurcations outside of this, in accordance with Eqs. (42) and (45). Perturbations of these conservative and purely dissipative cases are similar, but with asymmetrically located $\boldsymbol{Z}_{2}$-symmetric Bogdanov-Takens points, as seen for $\mu=0.04+0.2 i$ [panel (b)] and for $\mu=$ $0.2+0.04 i$ [panel (d)].

A more complete bifurcation picture is shown in Fig. 17 for the case of $a=0$ and $\mu=0.2 i$, when the primary Hopf bifurcation set is centered around $v=0$. We first note the 
broad similarities with Fig. 9. Primary periodic solutions are destroyed via saddle-node heteroclinic or (less often) heteroclinic bifurcation. The bifurcation structure is most complex for positive detuning where a series of saddle-node bifurcations leads to hysteresis and isolas of steady states. Again, there is a very complicated region near the cusp of saddle-node bifurcations at $(v, f)=(1.116,1.099)$, where a homoclinic bifurcation set arising from one BogdanovTakens point collides with the upper branch of saddle-node bifurcations, initiating a set of saddle-node homoclinic bifurcations before quickly detaching and moving towards another Bogdanov-Takens point on the lower saddle-node branch; see the inset of Fig. 9 for the detail of this scenario, which is not visible on the scale of Fig. 17.

The main difference between this case and that of $\phi=\pi$ is the transition from primary Hopf to a pair of pitchfork bifurcations for $|v| \gtrsim 1$. These transitions, the details of which are shown in Fig. 18, are associated with an additional three Bogdanov-Takens points, two with $\boldsymbol{Z}_{2}$ symmetry and one without. The leftmost transition is organized by a BogdanovTakens bifurcation with $\boldsymbol{Z}_{2}$ symmetry (the simplest " $s=1$ " case of [29], Sec. 9.5.3, and of [40]; see also [41]) that, in addition to the Hopf and pitchfork bifurcations, generates a heteroclinic bifurcation set. This heteroclinic bifurcation set soon collides with a curve of saddle-node bifurcations originating very near the same Bogdanov-Takens point (similar to the scenario seen in [39]).

The transition from Hopf to pitchfork bifurcation near $v=$ 1 [see Fig. 18(b)] is again organized by a Bogdanov-Takens bifurcation with $\boldsymbol{Z}_{2}$ symmetry, but according to the more complex case (the " $s=-1$ " case of [29], Sec. 9.5.3, and of [40]), which generates an additional set of asymmetric Hopf bifurcations, producing asymmetric periodic orbits $\left(P_{a}\right)$, and a set of homoclinic gluing bifurcations; a set of saddle-node bifurcations on the $\boldsymbol{Z}_{2}$-symmetric periodic orbits is not shown. The asymmetric Hopf and gluing bifurcation sets remain close as $f$ is decreased until the lower pitchfork bifurcation curve is crossed; at this point a gluing bifurcation is forbidden by the stability of the trivial $\left(\boldsymbol{Z}_{2}\right.$-symmetric) state and it splits into separate homoclinic and heteroclinic bifurcations (as occurs for the single oscillator case of Fig. 3). The homoclinic bifurcation set terminates in another (asymmetric) Bogdanov-Takens point located on the set of saddle-node bifurcations originating from the lower pitchfork bifurcation near this crossing. The heteroclinic bifurcation set merges with the saddle-node bifurcation set near this same point, creating a set of saddle-node heteroclinic bifurcations.

Four representative bifurcation diagrams are shown in Fig. 19 for this set of parameters and should be compared with those of Fig. 10. For $v=-1.5$ there is no Hopf bifurcation, but a pair of pitchfork bifurcations at $f=1.714$ and $f=1.888$, respectively. These are followed by a saddle-node bifurcation at $f=2.275$. Note, in comparison with Fig. 10, the splitting of the stable and unstable steady state branches (for large $f$ ) into two distinct pairs.

For $v=0$ the primary instability at $f=1.01$ is a Hopf bifurcation, which produces a stable $\boldsymbol{Z}_{2}$-symmetric periodic orbit that persists until a saddle-node heteroclinic bifurcation at $f=1.15$. This is followed by a second saddle-node bifurcation at $f=1.389$. (a)

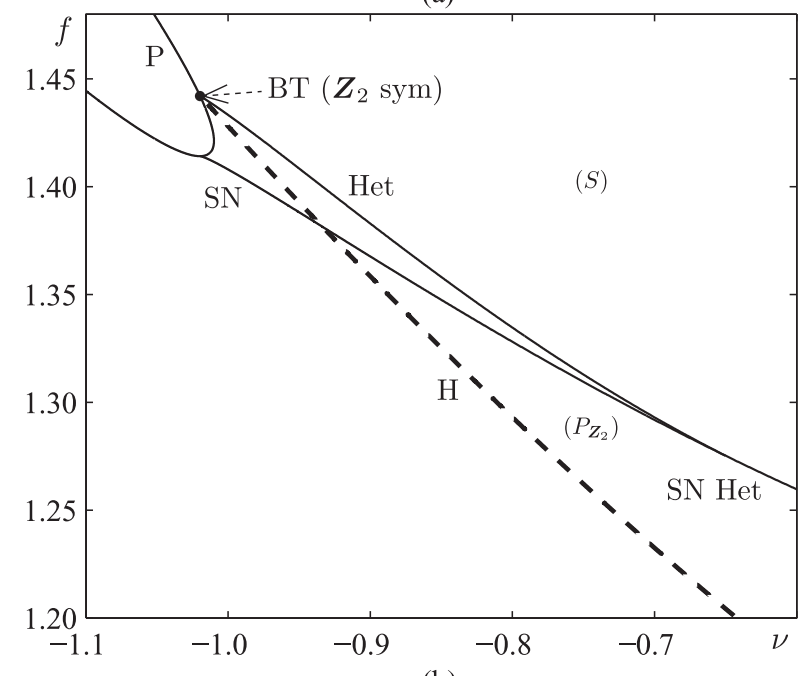

(b)

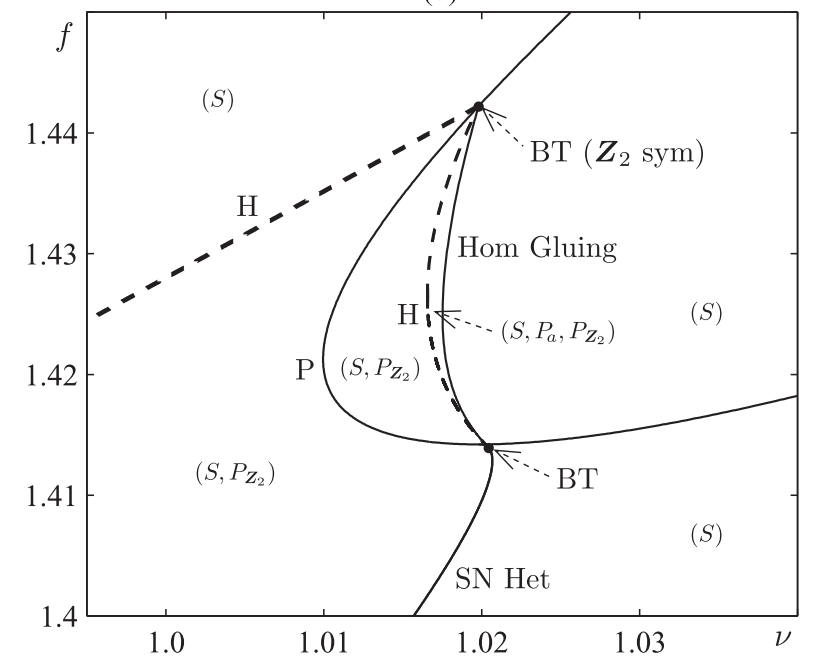

FIG. 18. Close-up of the (a) left and (b) right transition, between pitchfork and Hopf bifurcation of Fig. 17 showing bifurcation sets: pitchfork $(\mathrm{P})$, Hopf $(\mathrm{H}$, dashed curves), saddle-node (SN), saddlenode heteroclinic (SN Het), heteroclinic (Het), homoclinic gluing (Hom Gluing), and Bogdanov-Takens points (BT). Some bifurcation sets [homoclinic and heteroclinic, near the lower, asymmetric BT point in panel (b)] are not visible on the scale of the plot and are not labeled. In the larger regions, the types of solutions that exist there are indicated in parentheses.

For $v=1$ there is again a Hopf bifurcation, now at $f=$ 1.428 , but the unstable $\boldsymbol{Z}_{2}$-symmetric periodic orbit created here is soon destroyed in a saddle-node heteroclinic bifurcation at $f=1.374$ that forms the right boundary of an isola of steady states. The upper branch of this isola experiences a Hopf bifurcation [see the inset of Fig. 19(c)] at $f=1.076$, leading to a branch of stable asymmetric periodic orbits that is quickly destroyed by homoclinic collision with the lower branch at $f=1.0773$. A heteroclinic bifurcation near this point, at $f=$ 1.0848 , generates an unstable $\boldsymbol{Z}_{2}$-symmetric periodic orbit that undergoes a saddle-node bifurcation at $f=1.08461$, quickly followed by symmetry-breaking bifurcations at $f=1.08463$ and $f=1.08467$; the stabilized periodic orbit is eventually destroyed in another heteroclinic bifurcation at $f=1.121$ 


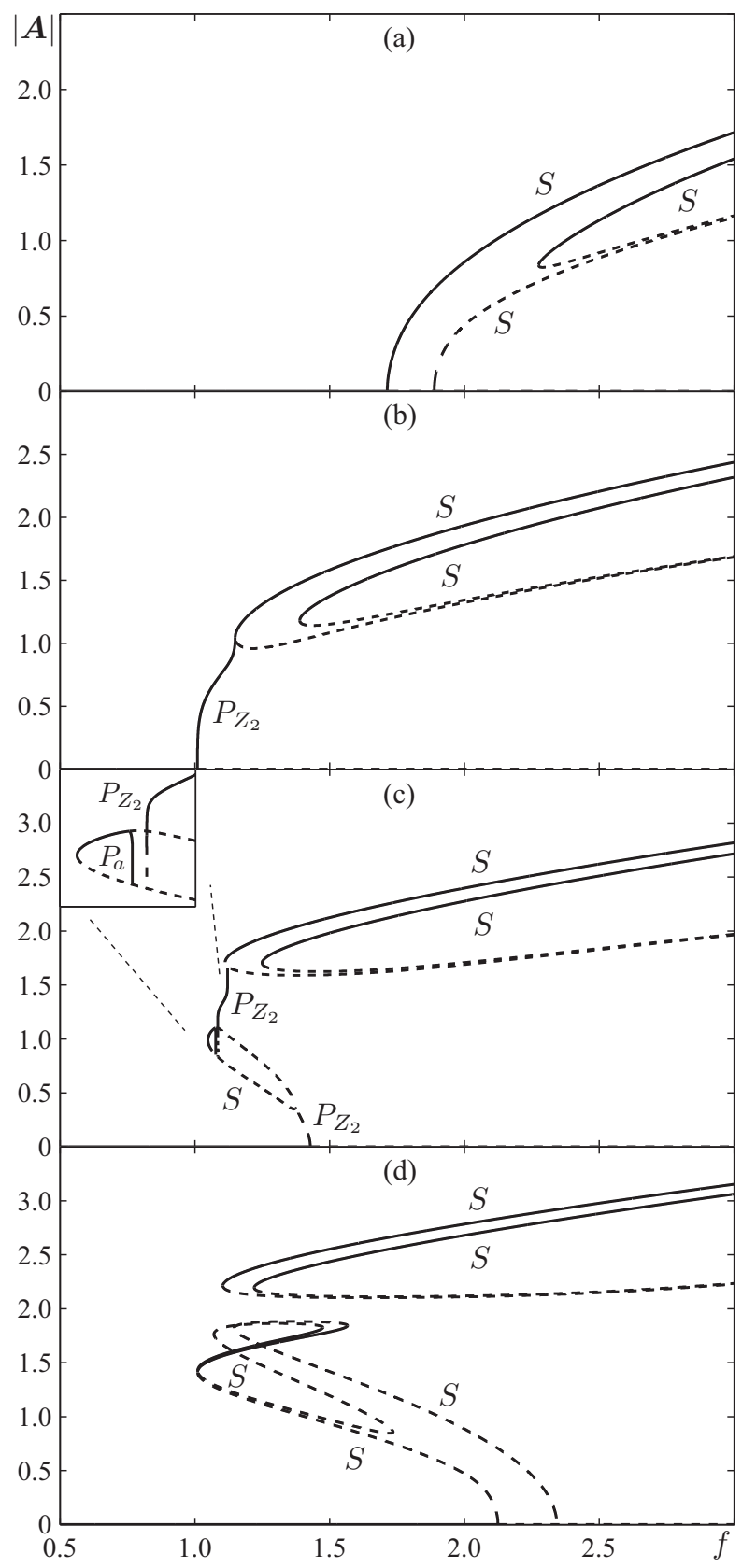

FIG. 19. Bifurcation diagrams for $\phi=\pi / 2, a=0, \mu=0.2 i$ showing the norm $|\boldsymbol{A}|=|(A, B)|$ of steady states $(S), \boldsymbol{Z}_{2}$-symmetric periodic orbits $\left(P_{Z_{2}}\right)$, and asymmetric periodic orbits $\left(P_{a}\right)$ : (a) $v=-1.5$, (b) $v=0$, (c) $v=1$, (d) $v=2$. For $v=1$ there is a secondary supercritical Hopf bifurcation on the isola of steady states that generates stable asymmetric periodic orbits, shown in the inset. Stable (unstable) solutions are shown with solid (dashed) lines.

involving a larger-amplitude steady state branch created via saddle-node bifurcation at $f=1.112$. The final saddle-node bifurcation occurs at $f=1.249$.

For $v=2$ the primary instability is again steady, with subcritical pitchfork bifurcations at $f=2.124$ and $f=2.343$. The first branch is stabilized in a saddle-node bifurcation at $f=1.01$, then destabilized again at $f=1.567$ before merging with the second branch in a third saddle-node bifurcation at $f=1.145$. An isola of steady states is present
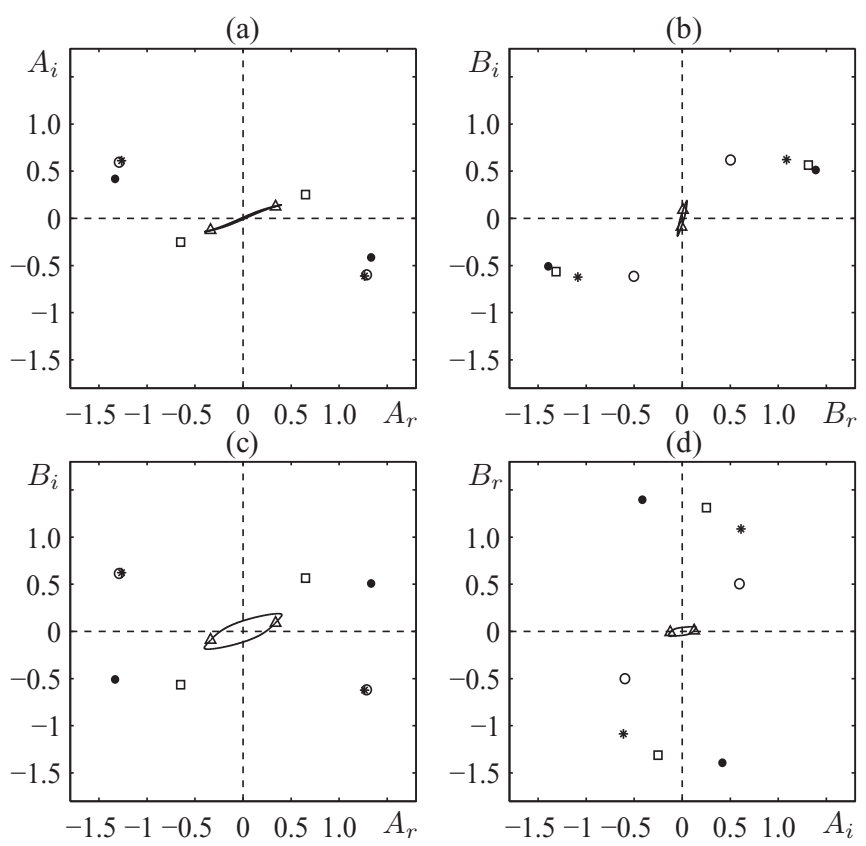

FIG. 20. Phase space portraits corresponding to the case of Fig. 19(c) with $f=1.374$ at the saddle-node heteroclinic bifurcation. There are two stable steady states (upper branch denoted by solid dots, and the lower branch by asterisks) and two (larger amplitude) unstable steady states (upper branch denoted by open circles, and the lower branch by open squares). The $\boldsymbol{Z}_{2}$-symmetric periodic orbit, which encloses the origin (trivial state not shown), is colliding with two smaller-amplitude unstable steady states (open triangles). Projections onto (a) $\left(A_{r}, A_{i}\right)$, (b) $\left(B_{r}, B_{i}\right)$, (c) $\left(A_{r}, B_{i}\right)$, and (d) $\left(A_{i}, B_{r}\right)$.

for $v=2$ as well. The stable portion of this isola exists between saddle-node bifurcations at $f=1.011$ and $f=1.475$, which mirror those bounding the stable branch described above. The unstable portion of the isola undergoes additional saddle-node bifurcations at $f=1.071$ and $f=1.735$. Finally, two more saddle-node bifurcations at $f=1.104$ and $f=1.22$ create the steady branches that persist for large $f$.

Phase portraits are shown in Fig. 20 for the case corresponding to Fig. 19(c) at $f=1.374$, which is where the $Z_{2}$-symmetric periodic orbit created in the primary Hopf bifurcation terminates in a saddle-node heteroclinic bifurcation. At this forcing value there are two stable steady state branches [each representing two solutions related by the reflection $R$ of Eq. (20)] and three unstable steady state branches. The smallest (pair) of these solutions is colliding with the periodic orbit. Note that the projections onto $\left(A_{r}, A_{i}\right)$ and $\left(B_{r}, B_{i}\right)$, which focus on the dynamics of one oscillator, are qualitatively similar to those of Fig. 11 (modulo a rotation of $B$ due to the forcing phase). The projections onto $\left(A_{r}, B_{i}\right)$ and $\left(A_{i}, B_{r}\right)$, however, which reflect the effects of coupling and symmetry, are quite different. The $\boldsymbol{C}_{4}$ symmetry of Figs. 11(c) and 11(d) is absent in Figs. 20(c) and 20(d).

\section{COMPARISON WITH EXPERIMENT}

Subharmonic surface waves in horizontally vibrated containers (see, e.g., [9,33,42-44]) are an important example of a real experimental system that can be modeled as a pair of 
(a)

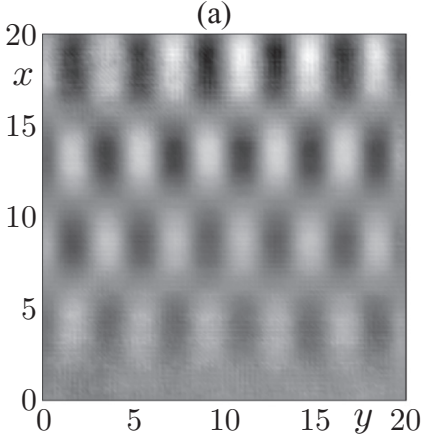

(c)
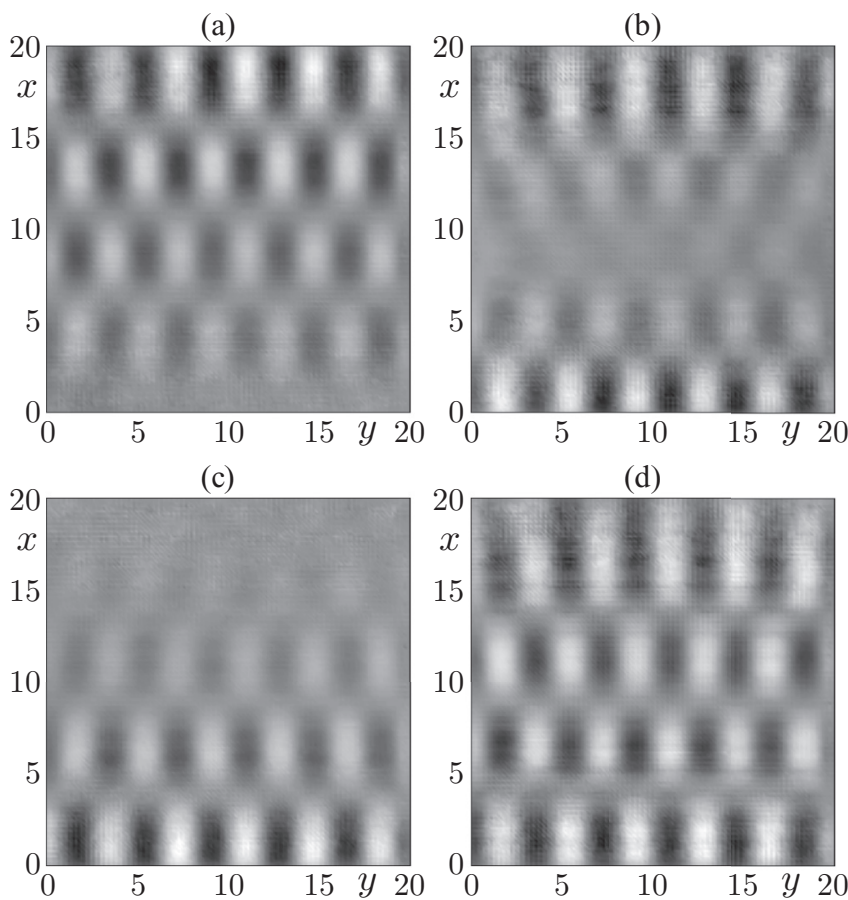

(d)

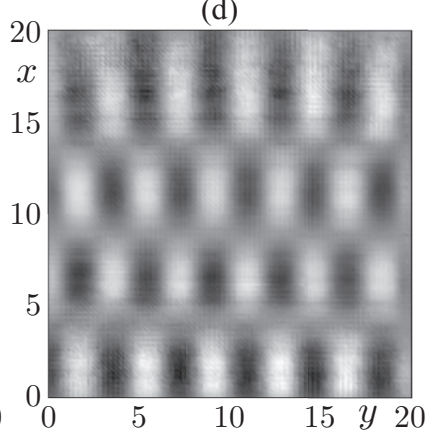

FIG. 21. Modulated (quasiperiodic) solution found experimentally in a 20-cm square container with $5 \mathrm{cSt}$ oil driven at $13.95 \mathrm{~Hz}$. The crosswise mode number is $N=11$ and the modulation period is about $45.6 \mathrm{~s}$. The snapshots (a)-(d) are separated by approximately $1 / 8$ of this period, or $5.7 \mathrm{~s}$. Reproduced from [20].

coupled parametrically forced oscillators with $\phi=\pi$. The case considered in detail in Sec. IV C 2 will be relevant if the subharmonic waves are dominant (i.e., the harmonic wave field can be neglected) and when the container is long enough (or forcing frequency high enough) that the subharmonic waves can be thought of as two weakly interacting (cross-wave) patterns generated by opposing endwalls. This is the case in several recent experiments $[19,20]$.

The results of Sec. IV C 2 agree with recent theoretical work on cross waves $[20,35,36,45]$ in predicting a primary Hopf bifurcation to modulated (quasiperiodic) solutions. The results of that section go further in, among other things, predicting the destruction of these modulated solutions in a saddle-node heteroclinic bifurcation (less often, in a standard heteroclinic bifurcation). Both the region of existence of these modulated solutions and their frequency of modulation decrease as the strength of the coupling decreases.

Although modulated solutions have been observed before in cross-wave experiments [44,46-48], these were likely due to either nonlinear effects or mode interactions, while the modulated solutions investigated here arise in a primary Hopf bifurcation and are due to interaction between the out-of-phase (by $\pi / 2$ ) wave fields generated at opposite sides of the container. These solutions, shown in Fig. 21, are associated with a slow cycling between balanced and one-sided excitation and were observed in the recent experiments of [20]. Possible reasons why they have not been more widely observed include the complicating effects of mode interactions mentioned above and the difficulty of controlling boundary conditions [19,20], but a more basic issue is simply selecting the right range of forcing frequencies for a given container. If the forcing frequency is too low, then cross waves will rapidly invade the entire surface and the interaction will be too strong (i.e., a weakly coupled oscillator model will not apply) and, among other difficulties, the distinction between one-sided and two-sided excitation is lost. If the forcing frequency is too high, then, although a weakly coupled oscillator approximation is valid, the modulation frequency will be too low to be measured accurately. Furthermore, the range of existence of these quasiperiodic solutions may be so narrow that it is washed out by various experimental errors.

Modulated solutions that cycle between one-sided and balanced excitation were located in the experiments of [20] using a $20-\mathrm{cm}$ square experimental container and a forcing frequency of 13-14 Hz. These solutions, located at onset, are an important confirmation of the modulated solutions predicted by theory. Additional details of Figs. 9 and 12, unfortunately, are impossible to verify due to the limitations of the measurement; there are substantial sources of experimental error, particularly in maintaining constant boundary conditions.

\section{A. Dependence on detuning}

A significant issue in comparing experiment with theory is the sensitivity to the coupling coefficient $\mu$. In the above analysis, this was treated as constant while forcing and detuning were varied, but in a real experiment it will not be so. Coupling strength depends on the overlapping wave fields and, thus, on all experimental parameters (container size, damping, boundary conditions, etc.). In particular, it varies strongly with detuning. Negative detuning leads to evanescent wave fields that decay quickly away from the endwall [20] (weak coupling), while positive detuning leads to more oscillatory wave fields that extend further into the interior (stronger coupling). Furthermore, the phase of the subharmonic waves in the overlap (coupling) region is important; some phases will lead to constructive interaction and others to destructive interaction [see Eq. (18)].

We thus expect that, for cross waves excited in horizontally vibrated containers, the coupling coefficient $\mu$ should grow with $v$ and oscillate as well, at least for $v>0$ when the wave fields are oscillatory in the interior. The oscillation with $v$ is clearly seen in the experimental measurements of the Hopf frequency, as well as theoretical models [20,35]; see Fig. 22.

As a first step to a more realistic dependence of the coupling coefficient $\mu(v)$, we use the following model,

$$
\mu(v)=\mu_{0} e^{\sigma v} e^{i\left[\theta_{0}+H(v) \Omega_{v} v\right]},
$$

where the exponent $\sigma$ captures the growth rate of the coupling due to increased wave penetration and $\Omega_{v}$ captures the oscillation due to varying overlap (phase). This oscillation is applied only for positive $v$ via a Heaviside step function $H(v)$.

The primary Hopf bifurcation and the corresponding Hopf frequency, obtained using Eq. (48), are shown in Fig. 23, which should be compared with Fig. 22 (reproduced from [20]). The particular parameters in Fig. 23 were chosen to qualitatively reproduce the experimental data (and theoretical curves) for the observed modulation frequency; Fig. 23 can be directly compared with Fig. 22 despite the different axes because of the rescaling (4) with $\gamma$ that is done here and not in [20] (for 


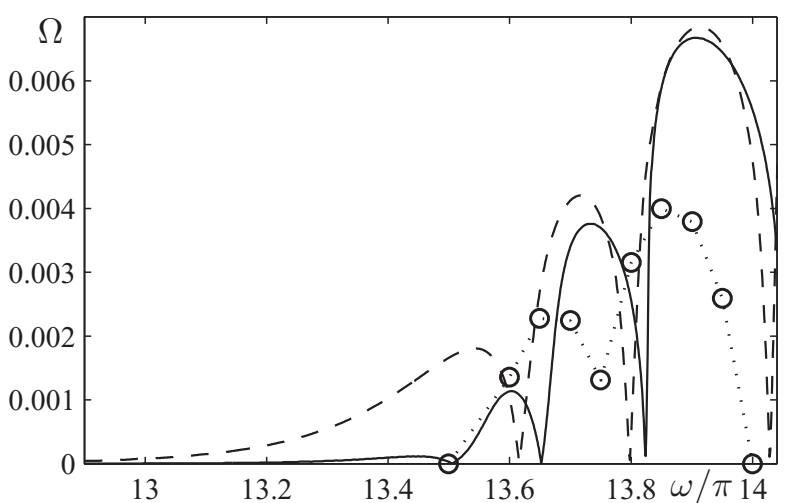

FIG. 22. Modulation (Hopf) frequency (in $\mathrm{Hz}$ ) of the solution shown in Fig. 21 over an interval of forcing frequencies $\omega / \pi$ (in $\mathrm{Hz}$ ) around the resonance frequency of $13.52 \mathrm{~Hz}$. The experimentally measured Hopf frequency $\Omega$ is compared with that predicted by the model obtained in [20] with the derived boundary conditions (solid curve) and with Dirichlet boundary conditions (dashed curve); remaining parameters for these calculations are viscosity, $5 \mathrm{cSt}$; surface tension, 19.7 dyne $/ \mathrm{cm}$; density, $0.913 \mathrm{~g} / \mathrm{cm}^{3}$; container depth, $5 \mathrm{~cm}$; and width, $9 \mathrm{~cm}$. Reproduced from [20].

that experiment $\gamma \sim 0.008)$. This choice of coupling function captures the oscillations of the modulation frequency $\Omega$ for positive $v$ seen in the experiment. Note also that, since the coupling $\mu(v)$ is small except for large detuning, the location of the Hopf bifurcation is only shifted with respect to the (pitchfork) instability of an uncoupled oscillator [dashed curve in Fig. 23(a)] by a small amount, most visible when $v$ is large and positive.

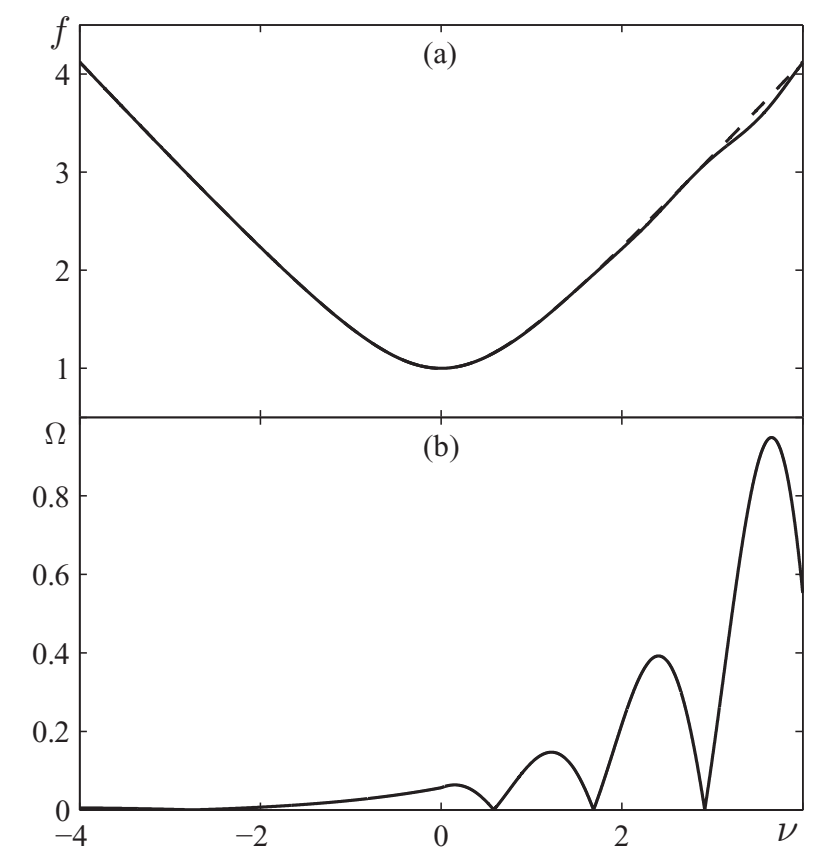

FIG. 23. Primary Hopf bifurcation for $\phi=\pi$ and $\mu(v)$ given by Eq. (48) with $\mu_{0}=0.06, \sigma=0.4, \theta_{0}=7 \pi / 18, \Omega_{v}=0.76 \pi$. (a) Critical forcing $f(v)$ (solid curve) compared to the pitchfork bifurcation of a single oscillator (dashed curve). (b) Hopf frequency $\Omega(v)$; this should be compared with Fig. 22 .

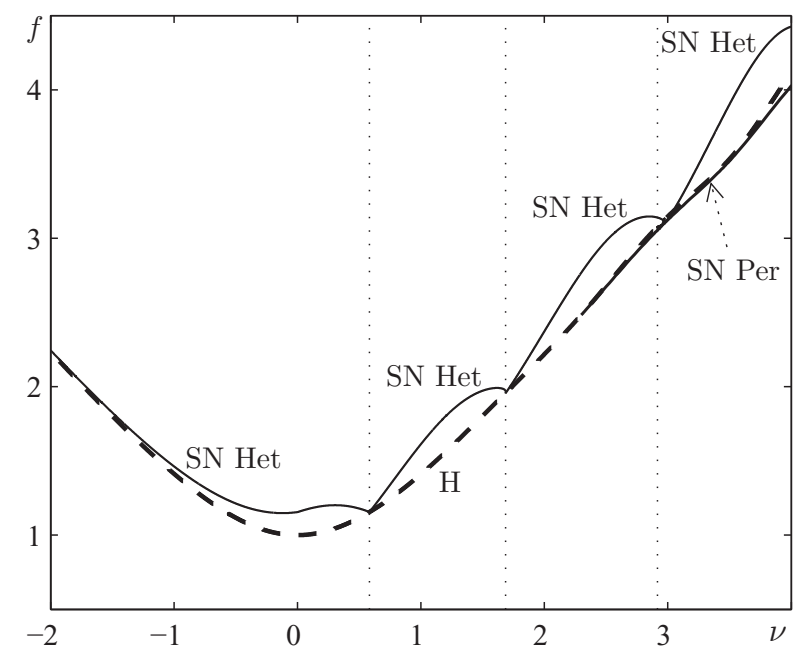

FIG. 24. Selected bifurcation sets for $\phi=\pi$ and $\mu(v)$ given by Eq. (48) with $\mu_{0}=0.06, \sigma=0.4, \theta_{0}=7 \pi / 18, \Omega_{v}=0.76 \pi$ : Hopf ( $\mathrm{H}$, dashed curve), saddle-node heteroclinic (SN Het), saddle-node of periodic orbits (SN Per, solid curves for $v \gtrsim 2.15$ ). Dotted vertical lines mark $v$ values where the Hopf frequency vanishes. Additional bifurcation sets near these double-zero points, which may include saddle-node, pitchfork, asymmetric Hopf, homoclinic, and heteroclinic bifurcations [38], are not shown in detail.

Additional bifurcation sets can also be calculated using the ansatz of Eq. (48) and compared with the experimental results. In particular, the experiments [20] show that stable modulated solutions exist well into the positive detuning regime. It is easy to show that this is not consistent with a conservative nonlinearity $a=0$ because the transition from supercritical to subcritical Hopf bifurcation occurs near $v=0$, and while stable periodic orbits can be found for larger $v$ they are soon destroyed by heteroclinic or saddle-node heteroclinic bifurcations; in Figs. 9 and 12 this occurs for $v \sim 1-1.5$, while experiments suggest stable modulated solutions are easily located for $v \sim 4$.

Figure 24 shows selected bifurcation sets calculated with $a=2$ and remaining parameters as in Fig. 23. Since $a=2$, the Hopf bifurcation is guaranteed to be supercritical for $v \leqslant 2$ (see Fig. 14), while for larger $v$ it can switch between supercritical and subcritical depending on the value of $\mu_{r}(v)$. The first two of these transitions with the parameters of Fig. 24 occur for $v \simeq 2.15$ (supercritical to subcritical) and $v \simeq 4.48$ (subcritical to supercritical); only the first of these is shown in the figure. Regardless of these transitions for $v>2$, there are substantial regions of stable modulated solutions, bounded above by a saddle-node heteroclinic bifurcation, for nearly all values of detuning, consistent with experiment.

\section{COMPARISON WITH SIMULATIONS}

Given the difficulties inherent in the experiments [20], it is not possible to accurately verify additional details of the dynamics beyond the initial Hopf bifurcation and the existence of a stable modulated solution, although some progress may be possible in future experiments. Here we take an alternative approach and compare with the results of direct numerical simulations [49] of the Navier-Stokes equations. These are 
done in a two-dimensional domain to make the calculations more feasible and, thus, cannot replicate the patterns observed in experiments, whose orientation is nearly crosswise. The simulations only permit one (downstream) orientation for the wave vector. These types of parallel subharmonic waves are known to exist [34-36], even if the instability is typically masked by the earlier onset of (nearly) crosswise oriented waves in the full three-dimensional problem.

In any case, the orientation of the subharmonic waves is incidental to the main idea examined here: that weakly coupled subharmonic patterns (more generally, parametrically forced oscillatory systems) driven by antisymmetric forcing, but otherwise identical, will reflect much of the dynamics contained in Eqs. (32). Although the values of the parameters in these equations are not known, we expect behavior similar to that shown in Fig. 10(a) or 10(b) for most frequencies, since this is the typical scenario for small detuning when the nonlinearity is dissipative, $a>0$.

The simulations described in this section were performed using fluid parameters similar to common silicone oils: viscosity of $5 \mathrm{cSt}$, surface tension of $20 \mathrm{dyn} / \mathrm{cm}$, and (for simplicity) density of $1 \mathrm{~g} / \mathrm{cm}^{3}$. The fluid domain was taken to be $7 \mathrm{~cm}$ long and $2.5 \mathrm{~cm}$ deep, and forcing frequencies (in the neighborhood) of 30, 40,50, and $60 \mathrm{~Hz}$ were used. A contact angle of $0^{\circ}$ was imposed on the lateral walls.

\section{A. Modulated solutions}

Figure 25 shows the filtered amplitude of the harmonic and subharmonic components taken from the times series of the height $h(t)$ of the left edge of the surface. Initial transient behavior is removed and the results are plotted versus forcing amplitude; the forcing was increased at a rate of $0.004 \mathrm{~g} / \mathrm{s}$ over the relevant part of the range (a faster rate was used over the initial portion safely below the subharmonic instability). Observe that for applied forcing at 30, 40, and $50 \mathrm{~Hz}$ the subharmonic instability appears to be subcritical, while the case of $60-\mathrm{Hz}$ forcing is less clear. Hysteresis is also seen using a slower rate of change, with both increasing and decreasing forcing (see Fig. 27). However, much of this observed hysteresis may be due to the delay effect of the (linear) drift in forcing [50,51], making it hard to distinguish the subcritical from the supercritical case.

With applied forcing at 30,40, and $50 \mathrm{~Hz}$, the subharmonic instability is oscillatory, and modulated solutions are found immediately after it. In each of these cases the period of modulation increases with forcing until the modulations abruptly disappear, evidence of a global bifurcation. For 30- and $40-\mathrm{Hz}$ forcing, there are additional transitions over the range of the simulation. With $30-\mathrm{Hz}$ forcing there is a second, more irregular, modulated state appearing at about $0.95 \mathrm{~g}$, while with $40-\mathrm{Hz}$ forcing there is an apparent transition to a second unmodulated branch of higher amplitude at about $1.49 \mathrm{~g}$.

The lack of an unambiguous region of modulated solutions with $60-\mathrm{Hz}$ forcing suggests this case may be close to a doublezero point where the Hopf frequency vanishes; see Fig. 24. This is discussed further below.

An instantaneous surface profile is shown in Fig. 26 for the case of $50-\mathrm{Hz}$ forcing and an amplitude of $0.9088 \mathrm{~g}$, which is above the onset of subharmonic waves. Note that
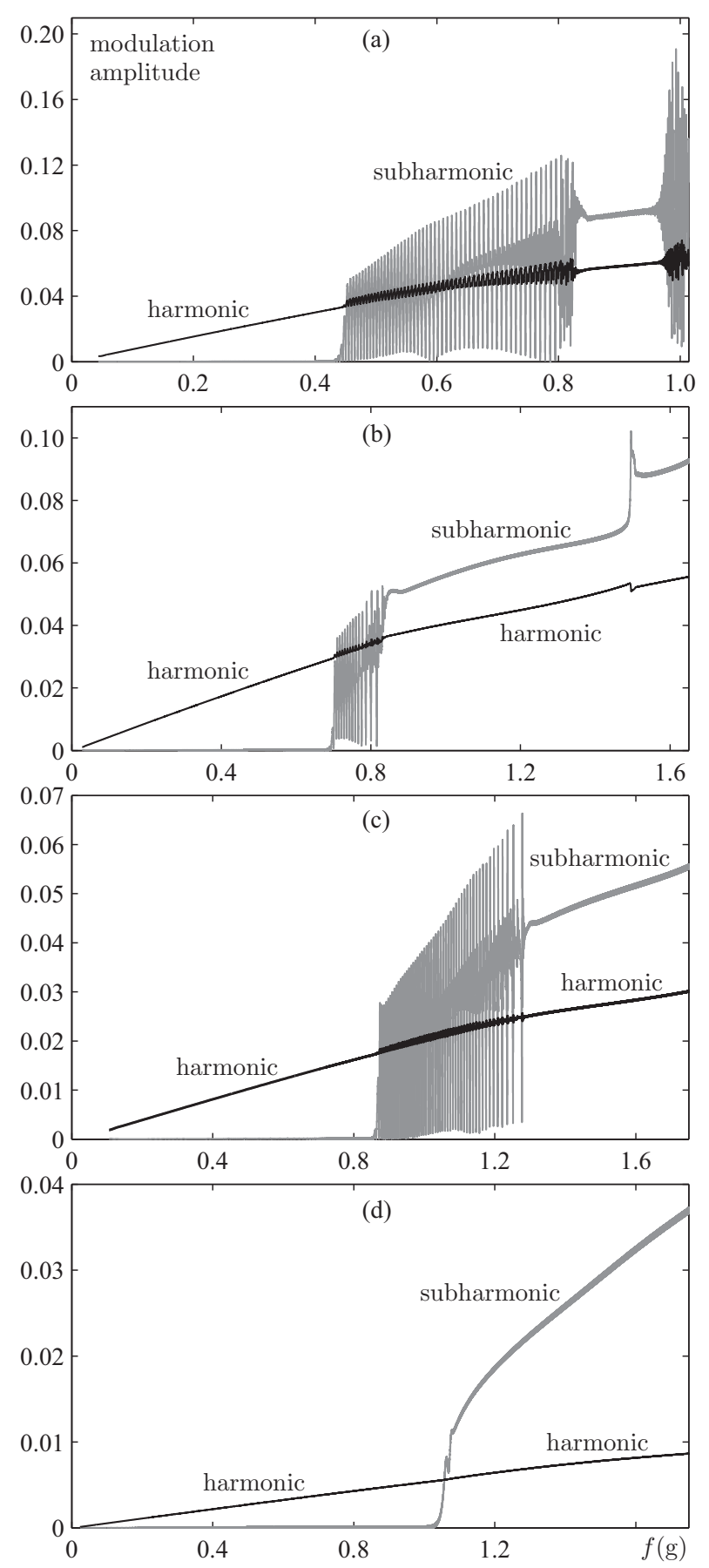

FIG. 25. Harmonic (black curve) and subharmonic (gray curve) components as forcing (in units of the gravitational acceleration $\mathrm{g}$ ) is increased for frequencies of (a) $30 \mathrm{~Hz}$, (b) $40 \mathrm{~Hz}$, (c) $50 \mathrm{~Hz}$, and (d) $60 \mathrm{~Hz}$. A transient has been removed from each simulation. Note the modulation of the subharmonic waves in all but (d) the $60-\mathrm{Hz}$ case.

the subharmonic waves dominate over the harmonic waves (in this snapshot, harmonic waves can be seen clearly only near the left endwall) and that the waves extend into the interior of the domain. A relatively short domain of $7 \mathrm{~cm}$ was intentionally chosen so that the modulated solutions would have a relatively large frequency and region of existence. A similar requirement was needed for the experimental observations [20]. It is perhaps surprising that a relatively strong interaction such as 


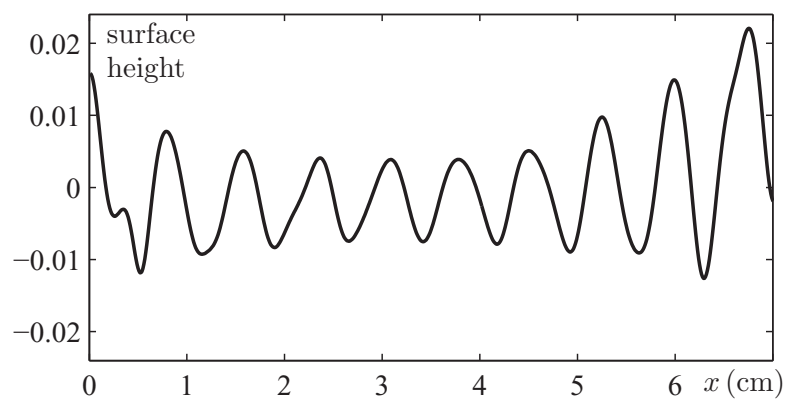

FIG. 26. Snapshot of the change in fluid surface height (in $\mathrm{cm}$ ) for the $50-\mathrm{Hz}$ simulation at $0.9088 \mathrm{~g}$. Above onset the subharmonic waves are dominant throughout the domain.

that in Fig. 26 can still be described by a model of weakly coupled parametrically forced oscillators.

\section{B. Character of the bifurcations}

We now examine the $50-\mathrm{Hz}$ case in more detail. Simulation results shown in Fig. 27(a) are obtained with a slower rate of change of applied acceleration $(0.0008 \mathrm{~g} / \mathrm{s})$ and in both increasing and decreasing directions. The Hopf transition still

(a)
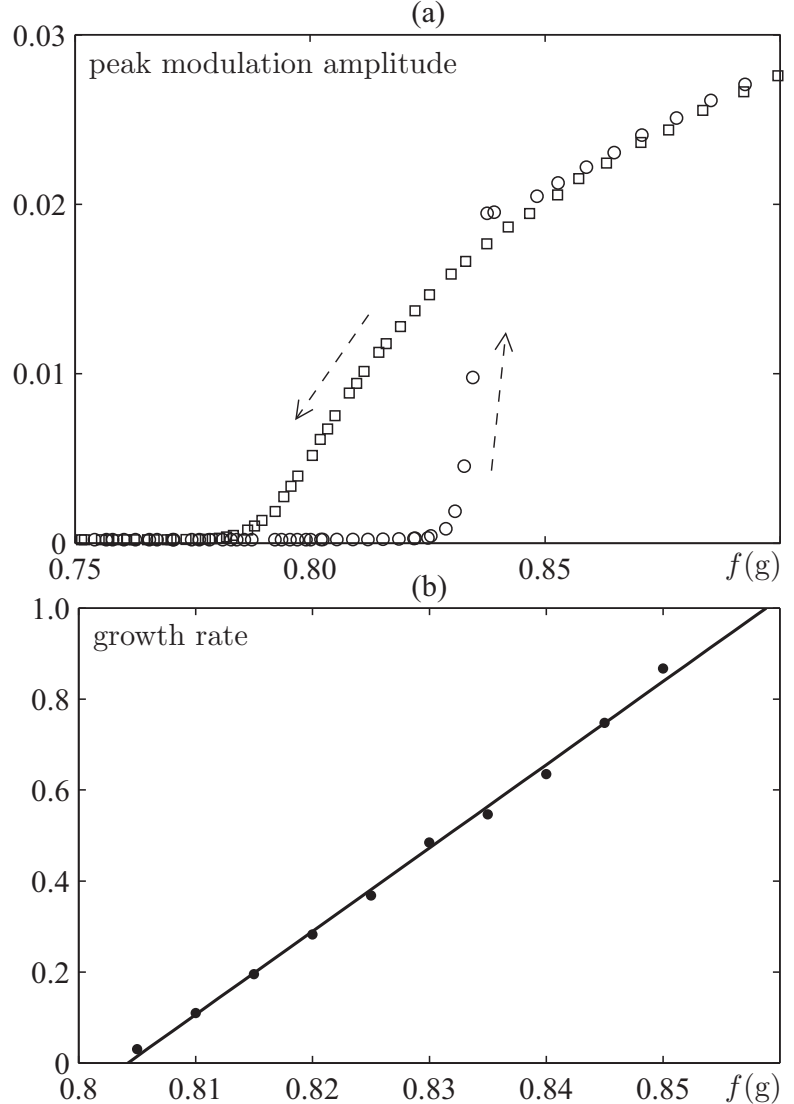

FIG. 27. (a) Close-up of the Hopf bifurcation to modulated subharmonic waves in the case of $50 \mathrm{~Hz}$ in Fig. 25(c). Peak modulation amplitude (maximum over one modulation period) of the filtered subharmonic signal is shown for increasing forcing (open circles, up arrow) and decreasing forcing (open squares, down arrow). (b) Linear fit of the growth rate (in $\mathrm{s}^{-1}$ ) of perturbations at fixed values of the forcing predicts an onset of $f=0.804 \mathrm{~g}$. appears to be hysteretic, although a measurement of the growth rate of perturbations at fixed forcing values suggests that the real location of the Hopf bifurcation is near $f=0.804 \mathrm{~g}$ and, thus, that much of this hysteresis is due to the linear drift in forcing [50,51].

As forcing amplitude is increased beyond the Hopf bifurcation, the modulated (quasiperiodic) solutions show an increase in modulation period and irregular (nonsinusoidal) temporal behavior indicative of global bifurcation. This progression can be seen in Fig. 28.

The increase in modulation period $\left(T_{m}\right)$ is shown quantitatively in Fig. 29 and compared with the inverse square root scaling expected of a saddle-node heteroclinic bifurcation. The excellent agreement argues that this is the bifurcation predicted in Eqs. (32) and not, for example, a standard heteroclinic bifurcation, which would show logarithmic divergence of $T_{m}$ (a much poorer fit to the data).

It should be acknowledged that the fit shown in Fig. 29, while convincing, is not a completely rigorous confirmation of the saddle-node heteroclinic bifurcation. In particular, if the system is "close" to a double-zero point (vanishing Hopf frequency), there may be additional complications, even in the reduced model (32), including small regions of (standard) heteroclinic bifurcations (or pitchfork bifurcations) that replace the saddle-node heteroclinic bifurcation [38]. These issues are further blurred by the delay associated with the slow drift in forcing. Regarding these concerns, we note that all simulations with fixed parameters are consistent with the picture suggested above. Steady (unmodulated) subharmonic solutions were only found above the global (saddle-node heteroclinic) bifurcation, while the modulated subharmonic solution appears to be the only (nontrivial) attractor below it. In other words, the stable unmodulated branch seems to disappear below the assumed saddle-node heteroclinic bifurcation, as expected. Furthermore, the delay associated with the drift in forcing is relatively minor in this case: Reducing the rate of change of the forcing from $0.004 \mathrm{~g} / \mathrm{s}$ to $0.0005 \mathrm{~g} / \mathrm{s}$ and considering both forward and backward sweeps only lowers the estimate for the bifurcation point by $1 \%-2 \%$. For these reasons (as well as technical numerical issues [49]) we did not attempt in the present paper a more rigorous bifurcation analysis using the Navier-Stokes simulations, following, e.g., the noninvasive control approach of [52]. This is left for future work.

\section{Dependence on frequency}

Significant dependence on frequency (detuning) is anticipated in these simulations for the reasons given in connection with Eq. (48). As the forcing frequency increases, the wave number selected by the dispersion relation increases too. Due to boundary constraints and the spatial dependence of the forcing $[20,34,36]$, this wave number (gradient of the phase) is not uniform in space and cannot simply follow the dispersion relation as it would in the unbounded (vertical) Faraday wave problem. Nonetheless, we expect it to increase with frequency, at least locally near the endwalls, and thus for the phase of the interaction in the interior region [i.e., the phase of the coefficient $\mu$ in Eqs. (32)] to vary with detuning. 


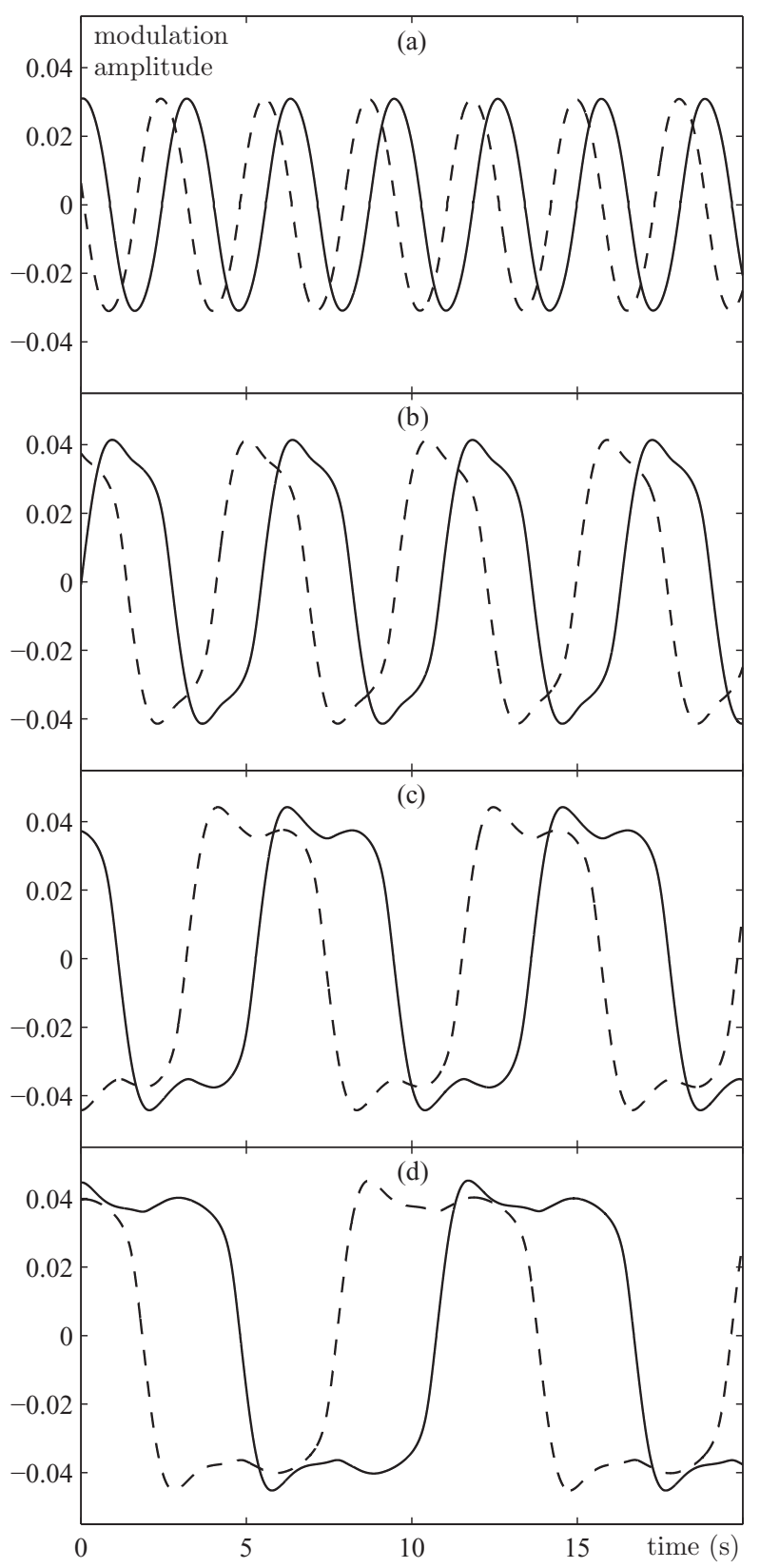

FIG. 28. Time series showing (signed) modulation amplitude for points equidistant from the left (solid curves) and right (dashed curves) boundaries for the case of $50 \mathrm{~Hz}$ forcing. The transition from sinusoidal modulation near the Hopf bifurcation to slower irregular modulation near the global bifurcation can be seen with increasing forcing values (in units of g): (a) 1, (b) 1.2, (c) 1.25, (d) 1.27. A 30-s transient has been removed from each simulation.

We can estimate the period of this variation using the gravity-capillary wave dispersion relation

$$
\omega^{2}=\mathrm{g} k+\frac{\Gamma}{\rho} k^{3},
$$

where $2 \omega$ is the forcing frequency (in $\mathrm{rad} / \mathrm{s}$ ), $\mathrm{g}$ is the gravitational acceleration, $\Gamma$ is the surface tension, and $\rho$ is the density. The wave number $k$ selected in the limit of small damping and forcing should satisfy $k_{n} \simeq n \pi / L$, where $L$ is the length of the container. These resonant modes are

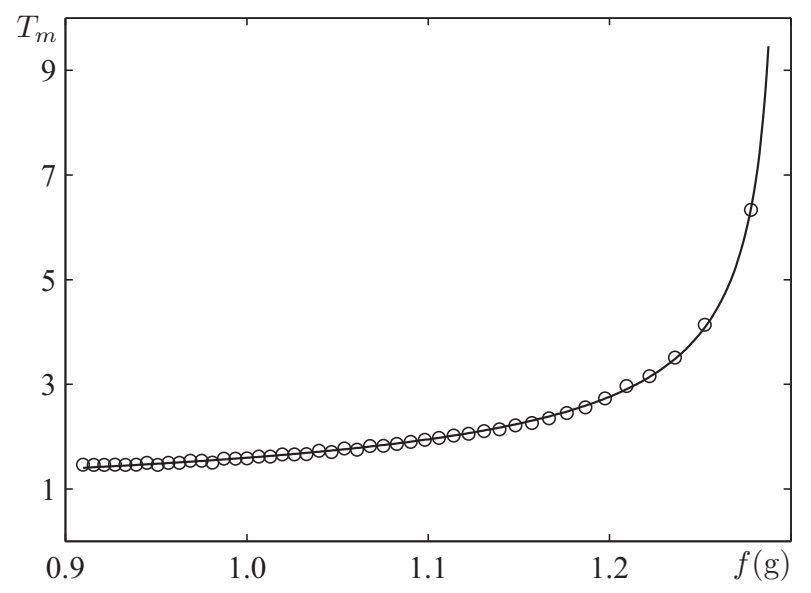

FIG. 29. The increase in modulation period $\left(T_{m}\right)$ as the global bifurcation is approached. The data from the simulation (open circles) fit well with the scaling expected for a saddle-node heteroclinic bifurcation: The solid line shows the fitting function, $0.059+$ $0.835(1.296-f)^{-1 / 2}$.

separated by several $\mathrm{Hz}$ for the parameters of the simulations (approximately $2.5 \mathrm{~Hz}$ near $40-\mathrm{Hz}$ forcing and $3 \mathrm{~Hz}$ near $60-\mathrm{Hz}$ forcing, e.g.). Thus, we expect the behavior of the fluid in the simulations to vary with frequency over a range of several $\mathrm{Hz}$.

First consider the case of $60 \mathrm{~Hz}$, where no clear region of modulated solutions is seen in Fig. 25. Figure 30 shows that this case is atypical; it just happens to be close to a double-zero point, where the region of existence of modulated solutions is very small (the dotted vertical lines in Fig. 24). With a forcing frequency of $60.4 \mathrm{~Hz}$, for instance, we observe modulated solutions terminating in a saddle-node heteroclinic bifurcation, as with $30-, 40-$, and $50-\mathrm{Hz}$ forcing.

The simulation has been repeated, using the same linear increase in forcing amplitude of $0.004 \mathrm{~g} / \mathrm{s}$, over a range of frequencies between 49 and $63.4 \mathrm{~Hz}$. The onset of subharmonic waves was estimated from the data, along with the initial modulation frequency $\Omega$, and the location of the saddle-node heteroclinic bifurcation where the modulations cease. It must be noted again that, due to the linear change (drift) in forcing amplitude and the finite time of the simulation, the threshold values will be somewhat overestimated. The results, which are shown in Fig. 31, verify the oscillations predicted above (with a period of about $3 \mathrm{~Hz}$ ) and are consistent

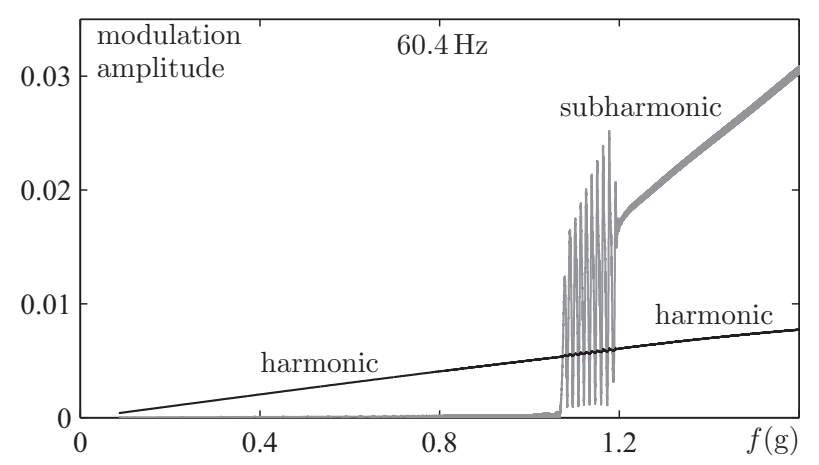

FIG. 30. Harmonic (black curve) and subharmonic (gray curve) components as forcing (in units of $\mathrm{g}$ ) is increased with $60.4-\mathrm{Hz}$ vibration. 


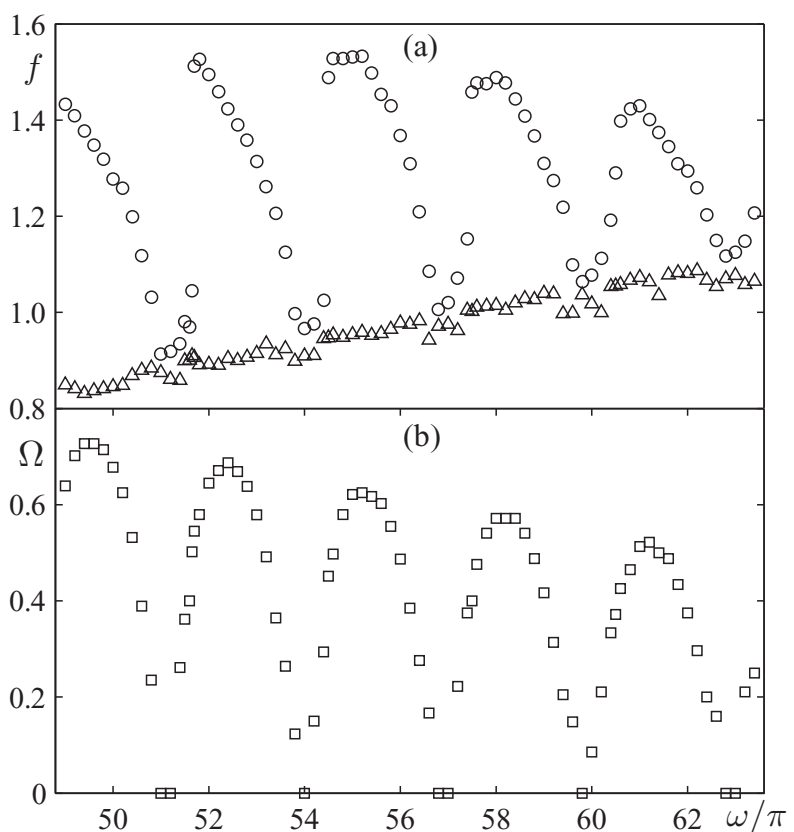

FIG. 31. (a) Estimated location of onset (triangles) and saddlenode heteroclinic bifurcation (circles); the parameters shown are applied forcing $f$ (in units of g) and applied frequency $\omega / \pi$ (in $\mathrm{Hz}$ ). (b) Estimated modulation frequency $\Omega$ (in $\mathrm{Hz}$ ) near onset.

with the picture provided in Fig. 24, where a sequence of double-zero points marks the boundaries of bubblelike regions where modulated solutions are found. Complex dynamics are expected in a neighborhood of these double-zero points [38]. The simulation results are further complicated by the mode transitions mentioned above. This adds a type of discontinuous behavior not captured by the weakly coupled parametric oscillator model analyzed in Sec. IV. Figure 31 suggests that these mode transitions occur just above the double-zero point (about $0.5 \mathrm{~Hz}$ higher in forcing frequency). The proximity of these two critical points and their associated complexity explains the irregular nature of the curves near the double-zero points.

\section{CONCLUSIONS}

In this paper we considered the dynamics of weakly coupled parametrically forced oscillators in a neighborhood of the dominant subharmonic instability. It was seen how different choices of coupling topology and individual forcing phases lead to distinct symmetry groups characterizing the equivariant bifurcation problem describing the primary instability. The residual permutation symmetry that survives in the coupled problem is critical in determining invariant subspaces and associated solution classes. It plays a key role as well in determining whether the primary bifurcation itself is steady or oscillatory.

Detailed results were obtained in the case of two coupled oscillators because of the relevance to recent experiments on horizontally vibrated fluids $[19,20]$. In horizontally forced fluid experiments there is a direct harmonic (synchronous) response including surfaces waves and localized evanescent modes. These evanescent modes (also, oscillatory bulk flow [34]) drive a variable pressure gradient that acts as a localized parametric forcing mechanism. The localized nature of the forcing and the resulting subharmonic modes means that, if the container is large compared to the spatial decay length of the waves, the subharmonic patterns generated at opposing endwalls can be treated as weakly interacting subharmonic oscillators.

The bifurcation sets and related bifurcation diagrams provided in Sec. IVC demonstrate the importance of the forcing phase in determining both primary and secondary instabilities. If the phase of the parametric forcing is identical for both oscillators, then the residual symmetry is $\boldsymbol{Z}_{2} \times \boldsymbol{Z}_{2}$ and there are two nontrivial fixed point subspaces containing synchronous (even) and antisynchronous (odd) oscillations. This structure, in particular, rules out a primary Hopf bifurcation.

If the parametric forcing is antisymmetric, then the residual symmetry is $\boldsymbol{C}_{4}$ and there are no nontrivial fixed point subspaces. The primary bifurcation is oscillatory and generates a $\boldsymbol{C}_{4}$-symmetric modulated (quasiperiodic) solution where the excitation cycles slowly between one oscillator and the other. These modulated solutions are destroyed via global bifurcation, which typically takes the form of a saddlenode heteroclinic connection, when the forcing exceeds the critical value by an $O(|\mu|)$ amount. A particularly complex interaction of saddle-node, Hopf, heteroclinic, and homoclinic bifurcations is found for positive detuning and associated with a pair of Bogdanov-Takens points.

If the phase difference between the parametric forcing at each oscillator is not 0 or $\pi$, then there is no residual permutation (exchange) symmetry, only the basic subharmonic symmetry $\boldsymbol{Z}_{2}$. This case leads to intermediate behavior organized by (generally two) $\boldsymbol{Z}_{2}$-symmetric Bogdanov-Takens points separating regions of primary Hopf and pitchfork bifurcations. As with antisymmetric forcing, a complex scenario is found for positive detuning and associated with additional (asymmetric) Bogdanov-Takens points.

The case of antisymmetric forcing was compared with the experimental results of [20] in Sec. V and found to qualitatively capture the observed oscillations in the modulation frequency if a physically motivated dependence of coupling on detuning is assumed. Regions of stable modulated solutions exist for a wide range of detuning if the nonlinearity is dissipative $(a>0)$. Further comparison with direct numerical simulations was presented in Sec. VI. For most parameters, the predicted modulated (quasiperiodic) solutions were found, and these were seen to terminate in a global bifurcation with increasing forcing. Furthermore, this was shown to be a saddle-node heteroclinic bifurcation, as expected from the coupled oscillator results of Sec. IV C 2. Oscillations with forcing frequency were expected in these two-dimensional simulations, due to both mode transitions and the phase of the coupling (interaction). The results were consistent with the scenario discussed in Sec. VA, where bubbles of stable modulated solutions are separated by double-zero points.

In summary, we have shown that coupled parametrically forced oscillators can have very interesting dynamics in the weakly nonlinear regime and that this dynamics is especially influenced by the residual permutation symmetry, if any, that remains. The fact that the underlying oscillators are parametric, with (at least) two preferred phases, also plays a key role in 
determining the relevant symmetries. Although simple models like the one considered here have obvious limitations, they allow a much more complete analysis and can shed light on complex behavior that would otherwise resist explanation. In this case, the relevance to cross-wave experiments was demonstrated by the symmetry-based explanation for the primary Hopf bifurcation, the presence of stable quasiperiodic solutions, the dependence of the modulation frequency on detuning, and the observation of the predicted saddle-node heteroclinic bifurcation in direct numerical simulations of the
Navier-Stokes equations. It is hoped that future experiments will allow more of the predictions of Sec. IV C 2 to be tested.

\section{ACKNOWLEDGMENTS}

This work was supported by the Ministerio de Ciencia e Innovación under Project No. AYA2010-19081 and the Ministerio de Economía y Competitividad under Projects No. ESP2013-45432-P and No. ESP2015-70458-P.
[1] P. Turchin, Complex Population Dynamics: A Theoretical/Empirical Synthesis (Princeton University Press, Princeton, NJ, 2003).

[2] N. Scafetta, J. Atmos. Sol.-Terr. Phys. 72, 951 (2010).

[3] C. Huygens, Phil. Trans. R. Soc. London 4, 937 (1669).

[4] R. E. Mirollo and S. H. Strogatz, SIAM J. Appl. Math. 50, 1645 (1990).

[5] Y. Kuramoto, Chemical Oscillations, Waves, and Turbulence (Springer-Verlag, Berlin, 1984).

[6] E. Buks and M. L. Roukes, J. Microelectomech. Syst. 11, 802 (2002).

[7] R. Lifshitz and M. C. Cross, Phys. Rev. B 67, 134302 (2003).

[8] P. B. Phua, K. S. Lai, R. F. Wu, and T. C. Chong, Opt. Lett. 23, 1262 (1998).

[9] J. Miles and D. Henderson, Annu. Rev. Fluid Mech. 22, 143 (1990).

[10] J. D. Lin and L. N. Howard, Hydrodynamics Laboratory Technical Report 44, M.I.T., 1960.

[11] L. F. McGoldrick, Tech. Rep. 2, University of Chicago, Department of Geophysical Sciences, 1968.

[12] B. J. S. Barnard and W. G. Pritchard, J. Fluid Mech. 55, 245 (1972).

[13] S. Taneda, Fluid Dyn. Res. 13, 119 (1994).

[14] F. Moisy, G.-J. Michon, M. Rabaud, and E. Sultan, Phys. Fluids 24, 022110 (2012).

[15] M. Funakoshi and S. Inoue, J. Fluid Mech. 192, 219 (1988).

[16] Z. C. Feng, Phys. Rev. Lett. 79, 415 (1997).

[17] M. Nobili, S. Ciliberto, B. Cocciaro, S. Faetti, and L. Fronzoni, Europhys. Lett. 7, 587 (1988).

[18] O. M. Faltinsen, O. F. Rognebakke, and A. N. Timokha, Phys. Fluids 18, 012103 (2006).

[19] J. Porter, I. Tinao, A. Laverón-Simavilla, and C. A. Lopez, Fluid Dyn. Res. 44, 065501 (2012).

[20] I. Tinao, J. Porter, A. Laverón-Simavilla, and J. Fernández, Phys. Fluids 26, 024111 (2014).

[21] I. Bena and C. Van den Broeck, Europhys. Lett. 48, 498 (1999).

[22] D. Goldobin and A. Pikovsky, Europhys. Lett. 59, 193 (2002).

[23] M. Copelli and K. Lindenberg, Phys. Rev. E 63, 036605 (2001).

[24] R. Kawai, K. Lindenberg, and C. Van den Broeck, Phys. A (Amsterdam, Neth.) 312, 119 (2002).

[25] P. M. Kitanov, W. F. Langford, and A. R. Willms, Dynam. Cont. Dis. Ser. A 20, 197 (2013).
[26] P. Danzl and J. Moehlis, Nonlinear Dyn. 59, 661 (2010).

[27] N. W. McLachlan, Theory and Application of Mathieu Functions (Clarendon Press, Oxford, U.K., 1947).

[28] T. B. Benjamin and F. Ursell, Proc. R. Soc. London, Ser. A 225, 505 (1954).

[29] Y. A. Kuznetsov, Elements of Applied Bifurcation Theory, 3rd ed., Applied Mathematical Sciences (Springer, Berlin, 2004).

[30] L. P. Shilnikov, Sov. Math. Dokl. 10, 1368 (1969).

[31] H. Arbell and J. Fineberg, Phys. Rev. E 65, 036224 (2002).

[32] M. Faraday, Philos. Trans. R. Soc. London 121, 299 (1831).

[33] A. F. Jones, J. Fluid Mech. 138, 53 (1984).

[34] F. Varas and J. M. Vega, J. Fluid Mech. 579, 271 (2007).

[35] J. M. Perez-Gracia, J. Porter, F. Varas, and J. M. Vega, J. Fluid Mech. 739, 196 (2014).

[36] J. Porter, I. Tinao, A. Laverón-Simavilla, and J. Rodríguez, Phys. Rev. E 88, 042913 (2013).

[37] Numerical computations were performed with XPPAUT (http://www.math.pitt.edu/ bard/xpp/xpp.html), AUTO-07P (http://sourceforge.net/projects/auto-07p/) and MATCONT (http://sourceforge.net/projects/matcont/).

[38] F. Marques, A. Meseguer, J. M. Lopez, J. R. Pacheco, and J. M. Lopez, Proc. R. Soc. London A 469, 20120348 (2013).

[39] L. S. Tuckerman, Fluid Dyn. Res. 44, 031202 (2012).

[40] G. Peng and C. Li, Int. J. Comput. Math. 91, 461 (2014).

[41] E. Knobloch, Phys. Lett. A 115, 199 (1986).

[42] C. J. R. Garrett, J. Fluid Mech. 41, 837 (1970).

[43] J. Miles, J. Fluid Mech. 186, 119 (1988).

[44] W. B. Underhill, S. Lichter, and A. J. Bernoff, J. Fluid Mech. 225, 371 (1991).

[45] J. M. Perez-Gracia, J. Porter, F. Varas, and J. M. Vega, Fluid Dyn. Res. 46, 041410 (2014).

[46] L. Shemer and S. Lichter, Phys. Fluids 30, 3427 (1987).

[47] S. Lichter and W. B. Underhill, Phys. Rev. A 35, 5282 (1987).

[48] H. Ayanle, A. J. Bernoff, and S. Lichter, Phys. D (Amsterdam, Neth.) 43, 87 (1990).

[49] COMSOL Multiphysics 3.5a, www.comsol.com.

[50] P. Mandel and T. Erneux, J. Stat. Phys. 48, 1059 (1987).

[51] S. M. Baer, T. Erneux, and J. Rinzel, SIAM J. Appl. Math. 49, 55 (1989).

[52] D. A. W. Barton and J. Sieber, Phys. Rev. E 87, 052916 (2013). 